\title{
Dynamic Catalysis Fundamentals: I. Fast calculation of limit cycles in dynamic catalysis
}

Brandon L. Foley, ${ }^{\mathrm{a}, *}$ and Neil K. Razdan ${ }^{\mathrm{b}}$

${ }^{a}$ Lawrence Livermore National Laboratory, 7000 East Avenue, Livermore, California 94550

${ }^{b}$ Department of Chemical Engineering and Materials Science, University of Minnesota, Twin Cities, Minneapolis, MN 55455

*Corresponding Author: E-mail: brfoley12@gmail.com

\section{Abstract}

Dynamic catalysis - the forced oscillation of catalytic reaction coordinate potential energy surfaces (PES) - has recently emerged as a promising method for the acceleration of heterogeneously-catalyzed reactions. Theoretical study of enhancement of rates and supraequilibrium product yield via dynamic catalysis has, to-date, been severely limited by onerous computational demands of forward integration of stiff, coupled ordinary differential equations (ODEs) that are necessary to quantitatively describe periodic cycling between PESs. We establish a new approach that reduces, by $\gtrsim 10^{8} \times$, the computational cost of finding the time-averaged rate at dynamic steady state (i.e. the limit cycle for linear and nonlinear systems of kinetic equations). Our developments are motivated by and conceived from physical and mathematical insight drawn from examination of a simple, didactic case study for which closed-form solutions of rate enhancement are derived in explicit terms of periods of oscillation and elementary step rate constants. Generalization of such closed-form solutions to more complex catalytic systems is achieved by introducing a periodic boundary condition requiring the dynamic steady state solution to have the same periodicity as the kinetic oscillations and solving the corresponding differential equations by linear algebra or Newton-Raphson-based approaches. The methodology is well- 
suited to extension to non-linear systems for which we detail the potential for multiple solutions or solutions with different periodicities. For linear and non-linear systems alike, the acute decrement in computational expense enables rapid optimization of oscillation waveforms and, consequently, accelerates understanding of the key catalyst properties that enable maximization of reaction rates, conversions, and selectivities during dynamic catalysis.

Keywords: Dynamic catalysis, limit cycles, asymmetric oscillation, analytical solution, fast calculations, linear algebra

\section{Introduction}

Experimental (1-6) and theoretical (7-10) reports have demonstrated that periodic input of thermodynamic work (e.g. by oscillation of applied electric potential) can effect orders-ofmagnitude improvement in catalytic turnover rates and overcome static equilibrium limits to chemical conversion akin to molecular motors/ratchets in biological systems $(11,12)$. So-called dynamic catalysis circumvents both kinetic and thermodynamic barriers by leveraging the kinetic asymmetry of two or more energetic states of the catalytic material to, for example, promote reactant adsorption and product desorption in a cyclic, stepwise fashion. In this sense, dynamic catalysis proffers a method to surpass static limits to turnover rates prescribed by the Sabatier principle by de-coupling and separately optimizing reactant and product binding energies, which are otherwise fundamentally interdependent.

The virtue of this technique has recently been demonstrated by calculation of rates and selectivities of various catalytic systems at dynamic steady state (i.e. the limit cycle). Dauenhauer and coworkers $(2,13)$ have shown that both simple three-step sequences and industrially-relevant reactions such as ammonia synthesis are, theoretically, profoundly accelerated by oscillation of the energetic state of the catalyst (e.g. by periodic oscillation of lattice strain). The current approach 
for calculating dynamic steady-state rates in such studies, however, primarily involves computationally expensive numerical forward integration of coupled ordinary differential equations (ODEs) until a limit cycle is reached. Simulation of reaction kinetics in this manner requires large calculation times that increase with oscillation frequency, requiring $\sim 1$ day for threestep reaction schemes at $10^{6} \mathrm{~Hz}$ and an expected $>300$ days for a frequency of $10^{10} \mathrm{~Hz}(14)$. These onerous computational demands hinder the exploration of vast parameter spaces that describe dynamic catalytic systems and, therefore, essentially proscribe discovery of the oscillation waveforms (shape and frequency) that maximize rate, yield, and/or selectivity.

In this work, we develop new strategies for the calculation of dynamic limit cycles disencumbered of the need to forward integrate stiff, coupled ODEs - the numerical solutions for which do not provide the mechanistic clarity characteristic of closed-form rate expressions. Our developments are informed by physical and mathematical intuition established from the examination of a model catalytic system, $\mathrm{A}+* \rightarrow \mathrm{A}^{*} \rightarrow \mathrm{B}+*$, oscillating between two kinetic states - each of which exclusively permits either $A+* \rightarrow A^{*}$ or $A^{*} \rightarrow B+*$. The simplicity of the two-step catalytic sequence allows for an exact analytical solution of dynamic steady-state rates and coverages solely in terms of elementary step rate constants and square waveform frequencies. The derived closed-form dynamic steady-state rate law reveals that (i) the optimal oscillation waveform is uniquely determined by elementary step rate constants, (ii) the optimal waveform may be asymmetric (e.g. more time is spent promoting $\mathrm{A}+{ }^{*} \rightarrow \mathrm{A}^{*}$ than $\mathrm{A}^{*} \rightarrow \mathrm{B}+{ }^{*}$ ), and (iii) the concept of catalytic resonance is not general; for the two-step catalytic reaction, rate is accelerated indefinitely with increase to oscillation frequencies.

The learnings from this didactic example are critically enabling in the development of linear algebra and Newton-Raphson based approaches that generalize analytical methods used to derive 
closed-form solutions and, in doing so, calculate the limit cycle for three-step reactions in milliseconds to seconds, $\gtrsim 10^{8} \times$ faster than previous methods (14). The expedience of the developed mathematical and algorithmic methods enables facile discovery of dynamic catalysis conditions that optimize both (i) the magnitude of oscillation of, for example, A* binding energy and (ii) the wavelength/duration of the oscillation in each energetic state. Linear algebra methods reveal that previously observed resonance regimes are defined by eigenvalues of the matrices that describe governing reaction ODEs; these eigenvalues formalize the concept of characteristic/resonance time scales of catalysis and, like in the two-step example, are relatable, in closed-form, to elementary step rate constants.

Complex reaction sequences proceeding via non-linear elementary steps (e.g. bimolecular surface reaction) are not fully describable by matrix algebra methods and, therefore, we instead recast the description of non-linear systems as an optimization problem solved by NewtonRaphson-based approaches. Formulation of non-linear catalytic reactions in the framework of mathematical optimization enables calculation and physical characterization of non-unique steady states that we surmise are intrinsic to non-linear reactions and therefore may hinder dynamic control of industrially-relevant reactions.

\section{Methods}

All functions and scripts are written in Octave GNU and Matlab ${ }^{\circledR} 2020 \mathrm{a}$. The code available to download for free from https: llwww.github.com/foley352/dynamic. Computational times are measured using the "tic" and "toc" functions. 


\section{Results and Discussion}

\subsection{Finding analytical solutions for limit cycles in dynamic catalysis}

We begin our discussion by considering the simplest kinetic system suitable for rate enhancement under dynamic catalysis conditions (Scheme 1), for which we will derive an analytical solution for the time-averaged rate at dynamic steady state. In Scheme 1, there are two reaction steps in series: the adsorption of $\mathrm{A}$ and the desorptive conversion of $\mathrm{A}^{*}$ to $\mathrm{B}$. We consider the case where there is a square-wave oscillation between two kinetic states, $\mathrm{j}$. Each kinetic state represents a different state of the catalyst (e.g., strain, electric potential) and has a different set of elementary step rate constants $k_{i}^{[j]}$, for state $j$ and elementary step $i$. During dynamic catalysis, the catalytic state, or potential energy surface (PES), oscillates with a wavelength $\lambda$ (or frequency $f=$ $1 / \lambda)$. In this example, the rate constants are $k_{i}=k_{i}^{[1]}$ for time $\delta t^{[1]}=\lambda / 2$ followed by $k_{i}=k_{i}^{[2]}$ for time $\delta t^{[2]}=\lambda / 2$, as illustrated in Figure 1a. This oscillation repeats indefinitely.

Without oscillation, the static steady-state rate, $r_{\mathrm{SS}}$, for the reaction network in Scheme 1 is $r_{\mathrm{SS}}=k_{1} k_{2} a_{\mathrm{A}} /\left(k_{1} a_{A}+k_{2}\right)$, which is zero for kinetic states 1 and 2 . Dynamic catalysis enables the coupling of these kinetic states to give a nonzero reaction rate, by first operating at kinetic state 1 to accumulate $\mathrm{A}^{*}$ on the surface and then switching to kinetic state 2 to convert $\mathrm{A} *$ to $\mathrm{B}$. Figure $1 \mathrm{~b}$ illustrates the oscillatory response of $\mathrm{A}^{*}$ coverage caused by the periodic switch between kinetic states 1 and 2 (Figure 1a). 

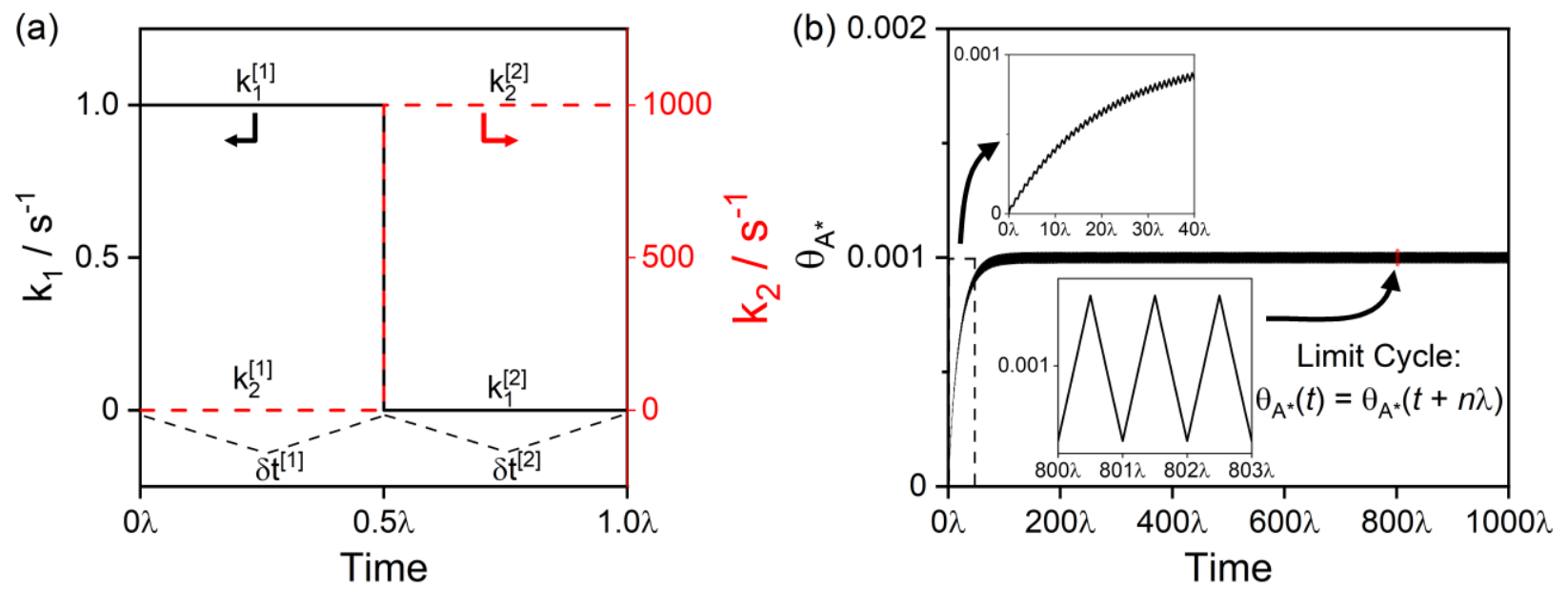

Figure 1. (a) Kinetic oscillation for $k_{1}$ and $k_{2}$. (b) Convergence to the limit cycle from a clean surface $\left(\theta_{*}=1\right)$ during dynamic catalysis for a symmetric oscillation between the kinetics states in Scheme 1 with $\lambda=10^{-4} \mathrm{~s}$ and $a_{\mathrm{A}}=1$.

The surface concentration history in Figure $1 \mathrm{~b}$ is determined by forward integration of the differential equation (eq. (1)):

$$
\frac{\mathrm{d} \theta_{\mathrm{A}^{*}}}{\mathrm{~d} t}=-k_{2}(t) \theta_{\mathrm{A}^{*}}+k_{1}(t) a_{\mathrm{A}} \theta_{*}=-k_{2}(t) \theta_{\mathrm{A}^{*}}+k_{1}(t) a_{\mathrm{A}}\left(1-\theta_{\mathrm{A}^{*}}\right)
$$

where $\theta_{*}+\theta_{\mathrm{A}^{*}}=1, k_{i}=k_{i}^{[1]}$ for $n \lambda \leq t<(n+1 / 2) \lambda$ and $k_{i}=k_{i}^{[2]}$ for $(n+1 / 2) \lambda \leq t<$ $(n+1) \lambda$, with the initial condition $\theta_{A^{*}}(t=0)=0$. After forward integration of hundreds of wavelengths, the fractional coverage of $A^{*}$ converges to a periodic limit cycle where (eq. (2)):

$$
\theta_{\mathrm{A}^{*}}(t)=\theta_{\mathrm{A}^{*}}(t+n \lambda)
$$

We contend that numerical forward integration (e.g. of eq. (1)), while quantitatively accurate, (i) does not provide the same physical insight or mathematical clarity as an analytical solution and (ii) is needlessly computationally intensive because the differential equations in dynamic catalysis are very stiff, and much of this computational cost is for calculating unnecessary information- 
the transient leading up to the limit cycle. In practice, we primarily are concerned with the behavior at the "dynamic steady state", which as shown in Figure 1b, is a limit cycle.

To this end, we establish a computationally efficient method for calculating the limit cycle for the reaction in Scheme 1 by finding the analytical solution to the limit cycle itself. Deriving the analytical solution is enabled by two key observations: (i) the differential equation in eq. (1) can be solved in piecewise fashion on the ranges from 0 to $\delta t^{[1]}$ and from $\delta t^{[1]}$ to $\delta t^{[1]}+\delta t^{[2]}$ because the rate constants are time-invariant over these ranges, and (ii) instead of an initial condition, as is used for forward integration, we can introduce continuity and periodic boundary conditions that satisfy the defining behavior of a limit cycle (eq. (2)). The analytical solution to eq. (1) in general is eq. (3):

$$
\theta_{\mathrm{A}^{*}}(t)=c_{j} \exp \left(-\left(k_{1}^{[j]} a_{\mathrm{A}}+k_{2}^{[j]}\right) t\right)+\frac{k_{1}^{[j]} a_{\mathrm{A}}}{k_{1}^{[j]} a_{\mathrm{A}}+k_{2}^{[j]}}
$$

where $c_{j}$ are the arbitrary constants of integration. Equation (3) collapses to the static steady-state solution for $t \rightarrow \infty$ in the absence of oscillation. Substituting $k_{i}^{[1]}$ and $k_{i}^{[2]}$ into eq. (3) gives the piecewise solution

$$
\theta_{\mathrm{A}^{*}}(t)=\left\{\begin{array}{cc}
c_{1} \exp \left(-k_{1}^{[1]} a_{\mathrm{A}} t\right)+1 & 0 \leq t<\delta t^{[1]} \\
c_{2} \exp \left(-k_{2}^{[2]}\left(t-\delta t^{[1]}\right)\right) & \delta t^{[1]} \leq t<\delta t^{[1]}+\delta t^{[2]}
\end{array}\right.
$$

where the $\left(t-\delta t^{[1]}\right)$ term is arbitrary and chosen for convenience when solving for the two unknown constants of integration, $c_{1}$ and $c_{2}$. The integration constants are determined by satisfaction of the continuity condition (eq. (5)):

$$
\lim _{t \rightarrow \delta t^{[1]^{-}}} \theta_{\mathrm{A}^{*}}(t)=\lim _{t \rightarrow \delta t^{[1]^{+}}} \theta_{\mathrm{A}^{*}}(t)
$$




$$
c_{1} \exp \left(-k_{1}^{[1]} a_{\mathrm{A}} \delta t^{[1]}\right)+1=c_{2}
$$

and the periodic boundary conditions (eq. (6)):

$$
\begin{aligned}
& \theta_{\mathrm{A}^{*}}(0)=\theta_{\mathrm{A}^{*}}\left(\delta t^{[1]}+\delta t^{[2]}\right) \\
& c_{1}+1=c_{2} \exp \left(-k_{2}^{[2]} \delta t^{[2]}\right)
\end{aligned}
$$

which ensure coverages are equal on either side of the switch from kinetic state 1 to 2 and from kinetic state 2 to 1 . The solution to the continuity and periodic boundary conditions gives (eq. (7)):

$$
\begin{aligned}
& c_{1}=\frac{1-\exp \left(-k_{2}^{[2]} \delta t^{[2]}\right)}{\exp \left(-k_{1}^{[1]} a_{\mathrm{A}} \delta t^{[1]}-k_{2}^{[2]} \delta t^{[2]}\right)-1} \\
& c_{2}=\frac{\exp \left(-k_{1}^{[1]} a_{\mathrm{A}} \delta t^{[1]}\right)-1}{\exp \left(-k_{1}^{[1]} a_{\mathrm{A}} \delta t^{[1]}-k_{2}^{[2]} \delta t^{[2]}\right)-1}
\end{aligned}
$$

Thus, we now have an analytical solution for $\theta_{\mathrm{A}^{*}}(t)$ after substitution of eq. (7) into eq. (4). The time-averaged rate during the limit cycle is defined as (eq. (8)):

$$
\begin{aligned}
& \langle r\rangle=\frac{\int_{0}^{\lambda} r(t) \mathrm{d} t}{\int_{0}^{\lambda} \mathrm{d} t}=\frac{\int_{0}^{\delta t^{[1]}} k_{2}^{[1]} \theta_{\mathrm{A}^{*}} \mathrm{~d} t+\int_{\delta t^{[1]}}^{\delta t^{[1]}+\delta t^{[2]}} k_{2}^{[2]} \theta_{\mathrm{A}^{*}} \mathrm{~d} t}{\delta t^{[1]}+\delta t^{[2]}} \\
& \langle r\rangle=\frac{\left(1-\exp \left(-k_{1}^{[1]} a_{\mathrm{A}} \delta t^{[1]}\right)\right)\left(1-\exp \left(-k_{2}^{[2]} \delta t^{[2]}\right)\right)}{\left(\delta t^{[1]}+\delta t^{[2]}\right)\left(1-\exp \left(-k_{1}^{[1]} a_{\mathrm{A}} \delta t^{[1]}-k_{2}^{[2]} \delta t^{[2]}\right)\right)}
\end{aligned}
$$

which, as expected, is a mathematically symmetric function (i.e. interchange of terms corresponding to states 1 and 2 gives an identical equation). The functional form of eq. (8) demonstrates that, unlike previously reported dynamic catalysis case studies, there is no effect of 
"catalytic resonance" (Figure 2a). The only condition relevant to rate enhancement for this system is whether the oscillation is sufficiently fast, such that $k_{1}^{[1]} a_{\mathrm{A}} \delta t^{[1]} \ll 1$ and $k_{2}^{[2]} \delta t^{[2]} \ll 1$. At these conditions, the surface coverage is approximately constant because the oscillation frequency is much faster than the time required for the surface coverages to change. We term this state the “quasi-static surface condition", at which eq. (8) simplifies to eq. (9):

$$
\langle r\rangle \approx \frac{k_{1}^{[1]} a_{\mathrm{A}} \delta t^{[1]} k_{2}^{[2]} \delta t^{[2]}}{\left(\delta t^{[1]}+\delta t^{[2]}\right)\left(k_{1}^{[1]} a_{\mathrm{A}} \delta t^{[1]}+k_{2}^{[2]} \delta t^{[2]}\right)}=\frac{k_{1}^{[1]} a_{\mathrm{A}} k_{2}^{[2]}\left(\frac{\delta t^{[2]}}{\delta t^{[1]}}\right)}{\left(1+\frac{\delta t^{[2]}}{\delta t^{[1]}}\right)\left(k_{1}^{[1]} a_{\mathrm{A}}+k_{2}^{[2]}\left(\frac{\delta t^{[2]}}{\delta t^{[1]}}\right)\right)}
$$

At quasi-static surface conditions, the rate of the reaction in Scheme 1 depends solely on the ratio $\delta t^{[2]} / \delta t^{[1]}$, with time-averaged rates shown in Figure $2 \mathrm{~b}$.

Scheme 1. Simplest dynamic catalysis reaction network.

\begin{tabular}{c|c}
\hline $\mathrm{A}+* \rightarrow \mathrm{A}^{*}$ & $k_{1}^{[1]}=1 \mathrm{~s}^{-1}$ \\
& $k_{1}^{[2]}=0 \mathrm{~s}^{-1}$ \\
& $k_{2}^{[1]}=0 \mathrm{~s}^{-1}$ \\
$\mathrm{~A}^{*} \rightarrow \mathrm{B}+*$ & $k_{2}^{[2]}=1000 \mathrm{~s}^{-1}$ \\
& \\
\hline Overall: $\mathrm{A} \Rightarrow \mathrm{B}$ & \\
\hline
\end{tabular}



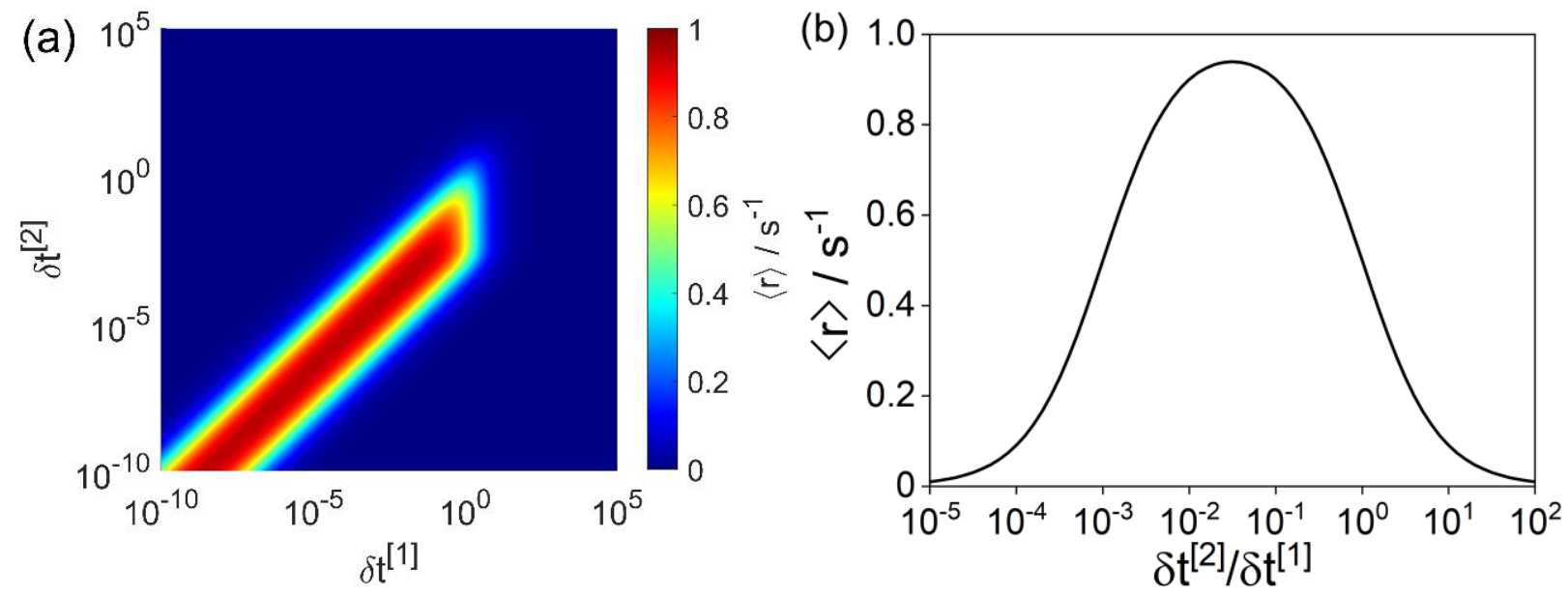

Figure 2. (a) Contour plot of the time-averaged rate as a function of $\delta t^{[1]}$ and $\delta t^{[2]}$ for the reaction in Scheme 1 with $a_{\mathrm{A}}=1$. (b) Time-averaged rate as a function of $\delta t^{[2]} / \delta t^{[1]}$ at quasi-static surface conditions.

Examination of eq. (9) reveals that, in general, the optimal ratio of $\delta t^{[2]} / \delta t^{[1]}$ is $\sqrt{k_{1}^{[1]} / k_{2}^{[2]}}$, as is evidenced by maximum rate occurring for $\delta t^{[2]} / \delta t^{[1]}=10^{-1.5}$ (Fig. 2 b). The discovery of this simple, consequential mathematical relationship is made possible by the analytical solution and demonstrates that (i) synergistic asymmetry in rate constants and oscillation waveform is key in determining the optimality of rate enhancement and (ii) the phenomenon of catalytic resonance frequency is not a general, or defining, feature of dynamic catalysis. In addition to the proffered physical insight, the analytical solution greatly reduces the computational time compared to forward integration. In the following, we generalize the presented analytical technique by development of an algorithmic procedure for reactions of any number of steps, network connectivity, and kinetic oscillation shape to programmatically find the time-averaged rates during dynamic catalysis. 


\subsection{A programmatic method for solving for dynamic catalysis limit cycles for linear reaction schemes}

In reaction schemes that do not involve the reaction between two species with timedependent concentrations, the coupled differential equations that describe the dynamics of fractional coverages are written in matrix form as (eq. (10)):

$$
\frac{\mathrm{d}}{\mathrm{d} t} \boldsymbol{\theta}=\boldsymbol{A}(t) \boldsymbol{\theta}
$$

where $\boldsymbol{\theta}$ is a vector of all surface species (including vacant sites) and $\boldsymbol{A}$ is a time-dependent matrix that is a function of rate constants and chemical activities of reactants and products, which are the coefficients that multiply the fractional coverages in each differential equation. Equation (10) closely resembles a system of coupled first-order ordinary differential equations, with two exceptions: (1) the coefficient matrix $\boldsymbol{A}$ is a function of time and (2) at any time, $\boldsymbol{A}$ is a singular (non-invertible) matrix because the fractional coverages are not linearly independent. To resolve the second issue, we must eliminate one of the fractional coverages by substituting $\theta_{j^{*}}=1-$ $\sum_{m^{*} \neq j^{*}} \theta_{m^{*}}$, which is equivalent to the following procedure: (1) remove the $j^{\text {th }}$ row of $\boldsymbol{A}$ and $\boldsymbol{\theta}$, (2) remove the $j^{\text {th }}$ column of $\boldsymbol{A}$ and rename it as a column vector $\boldsymbol{b}$, and (3) subtract $\boldsymbol{b}$ from each column of $\boldsymbol{A}$. The new matrix, $\boldsymbol{A}^{\prime}$, has one less row and column than $\boldsymbol{A}$ and is no longer singular. The new form of the coupled differential equations is (eq. (11)):

$$
\frac{\mathrm{d}}{\mathrm{d} t} \boldsymbol{\theta}^{\prime}=\boldsymbol{A}^{\prime}(t) \boldsymbol{\theta}^{\prime}+\boldsymbol{b}(t)
$$

where $\boldsymbol{\theta}^{\prime}$ is $\boldsymbol{\theta}$ with the $j^{\text {th }}$ row removed. Next, it is necessary to eliminate the time-dependence of $\boldsymbol{A}^{\prime}$ and $\boldsymbol{b}$. This is accomplished by discretizing continuous waves into square waves with $n$ steps. At the limit of $n \rightarrow \infty$, the $n$-stepped square wave converges to the continuous wave, as illustrated in Figure 3. On each of the flat terraces of the $n$-stepped square wave, the rate constants are not 
functions of time, and only change at the locations of the step discontinuities. Thus, for an $n$ stepped square wave, equation (11) can be rewritten as $n$ equations:

$$
\frac{\mathrm{d}}{\mathrm{d} t} \boldsymbol{\theta}^{[j]}=\boldsymbol{A}^{[j]} \boldsymbol{\theta}^{[j]}+\boldsymbol{b}^{[j]} \forall t \in\left[t^{[j-1]}, t^{[j]}\right]
$$

where the superscript $[j]$ refers to the $j^{\text {th }}$ step of the $n$-stepped square wave, and the primes (" ' ") have been dropped for clarity. There is one eq. (12) for each step of the square wave, and each equation is valid from the end of the previous step $\left(t^{[j-1]}\right)$ to the end of the present step $\left(t^{[j]}\right)$. The utility of formulating dynamic catalytic systems in terms of eq. (12) is that the coefficient matrix $\boldsymbol{A}^{[j]}$ and the vector $\boldsymbol{b}^{[j]}$ are not functions of time, and thus eq. (12) is in the form of a differential equation that is easily solved with linear algebra. The general solution to eq. (12) is of the form (eq. (13)):

$$
\theta_{m^{*}}^{[j]}(t)=p_{m^{*}}^{[j]}+\sum_{s=1} c_{s}^{[j]} v_{s_{m^{*}}}^{[j]} \exp \left(\lambda_{s}^{[j]}\left(t-t^{[j-1]}\right)\right) \forall t \in\left[t^{[j-1]}, t^{[j]}\right]
$$

where $\theta_{m^{*}}^{[j]}$ is the row of vector $\boldsymbol{\theta}^{[j]}$ corresponding to species $m^{*}, \boldsymbol{p}^{[j]}$ is the particular solution vector for the $j^{\text {th }}$ step, $c_{s}^{[j]}$ is the $s^{\text {th }}$ constant of integration in the $j^{\text {th }}$ step, and $\boldsymbol{v}_{\boldsymbol{s}}^{[j]}$ is the $s^{\text {th }}$ eigenvector of $\boldsymbol{A}^{[j]}$ with the corresponding eigenvalue $\lambda_{s}^{[j]}$. Subtraction of $t^{[j-1]}$ from $t$ in the exponential of eq. (13) is arbitrary and chosen for convenience such that the exponentials all equal unity at $t=t^{[j-1]}$. The solution presented in eq. (13) assumes no repeat eigenvalues of $\boldsymbol{A}^{[j]}$ and includes the particular solution to $\frac{d}{d t} \boldsymbol{\theta}^{[j]}=\mathbf{0}$, found by eq. (14):

$$
\boldsymbol{p}^{[j]}=-\left(\boldsymbol{A}^{[j]}\right)^{-1} \boldsymbol{b}^{[j]}
$$

The only remaining unknowns in eq. (13) are the integration constants $c_{s}^{[j]}$, which are found by satisfying the boundary conditions analogously to the two-step reaction in Scheme 1. For a system 
with $n$ steps in the square wave and $m$ surface species, there are $n \times(m-1)$ boundary conditions (e.g. for the reaction in Scheme 2, the number of boundary conditions is $2 \times(2-1)=2$ ).

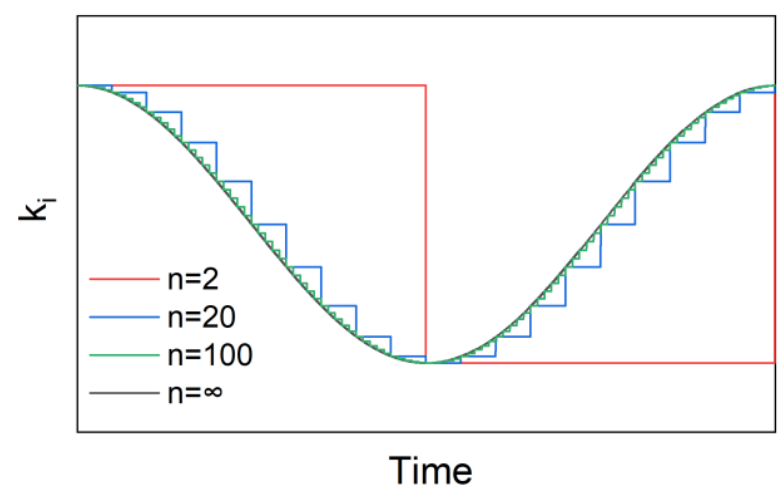

Figure 3. Discretization of a continuous sinusoidal wave into an $n$-stepped square wave. The approximation of the continuous wave improves with increasing $n$.

The boundary conditions for a dynamic catalytic system operating at the limit cycle are illustrated in Figure 4. The fractional coverages of all surface species must be continuous in time, which in vector form is written as (eq. (15)):

$$
\begin{gathered}
\boldsymbol{\theta}^{[j]}\left(t=t^{[j]}\right)=\boldsymbol{\theta}^{[j+1]}\left(t=t^{[j]}\right) \forall j<n \\
\boldsymbol{\theta}^{[n]}\left(t=t^{[n]}\right)=\boldsymbol{\theta}^{[1]}\left(t=t^{[0]}\right)
\end{gathered}
$$

where $n$ is the total number of steps and $t^{[0]}$ is the starting time for kinetic state 1 in the limit cycle (see Figure 4). For the last step (step 3 in Figure 4), there is no " $j+1$ " step after, and thus a periodic boundary condition is applied here requiring that the final fractional coverages in step $n$ are equal to the initial coverages in step 1 . We emphasize that the periodic boundary conditions in eq. (15) assume that the solution $\boldsymbol{\theta}(t)$ has the same periodicity as the initial coefficient matrix $\boldsymbol{A}(t)$ and discuss the existence of solutions that are aperiodic or that have different periodicities at the end of this section. 


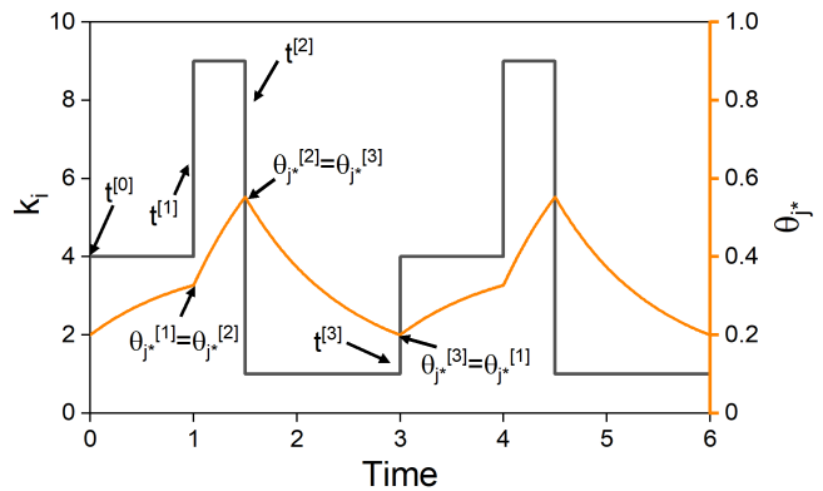

Figure 4. Illustration of the periodic boundary conditions imposed on fractional coverages during dynamic catalysis. The kinetic oscillation has a period of three time units, and the figure shows two periods.

By substitution of eq. (13) into (15), the boundary conditions can be written in the form of algebraic equations that are linear in the unknowns, $c_{s}^{[j]}$ (eq. (16)):

$$
\begin{gathered}
\sum_{s=1} c_{s}^{[j]} v_{s_{m^{*}}}^{[j]} \exp \left(\lambda_{s}^{[j]}\left(t^{[j]}-t^{[j-1]}\right)\right)-\sum_{s=1} c_{s}^{[j+1]} v_{s_{m^{*}}}^{[j+1]}+p_{m^{*}}^{[j]}-p_{m^{*}}^{[j+1]}=0 \forall j<n \\
\sum_{s=1} c_{s}^{[n]} v_{s_{m^{*}}}^{[n]} \exp \left(\lambda_{s}^{[n]}\left(t^{[n]}-t^{[n-1]}\right)\right)-\sum_{s=1} c_{s}^{[1]} v_{s_{m^{*}}^{[1]}}^{[1]} p_{m^{*}}^{[n]}-p_{m^{*}}^{[1]}=0
\end{gathered}
$$

Equation (16) represents a system of linear equations of the form given in eq. (17):

$$
M c+p=0
$$

where $\boldsymbol{c}$ is a vector of all $c_{s}^{[j]}, \boldsymbol{M}$ is a matrix of coefficients, and $\boldsymbol{p}$ is the vector of $p_{m^{*}}^{[j]}-p_{m^{*}}^{[j+1]}$, where each row in $\boldsymbol{M}$ and $\boldsymbol{p}$ corresponds to a different equation in eq. (16). Solving eq. (17) is often the slowest computational step for solving the limit-cycle fractional coverages with $n$-step square waves, and the computational cost of this step is essentially independent of oscillation frequency. With the constants of integration solved for, we can now describe the entire time- 
dependence of each species during the limit cycle. The time-averaged rates are found by analytical integration of the rate as a function of time (eq. (18)):

$$
\langle r\rangle=\frac{\int_{0}^{\lambda} r(t) \mathrm{d} t}{\lambda}
$$

Ardagh et al. (14) investigated the kinetics of the reaction in Scheme 2 with dynamic kinetics where the binding energy of surface species $\mathrm{B}^{*}$ is oscillated, and this binding energy correlates linearly with the (i) transition state energy for the A* to B* reaction and (ii) the binding energy of A* via Brønsted-Evans-Polanyi relations. The relationship between the binding energies is given by (eq. (19)):

$$
\mathrm{BE}_{\mathrm{A}}=\left(\mathrm{BE}_{\mathrm{B}}-(1-\gamma) \delta-\Delta H_{\mathrm{ovr}}\right) / \gamma
$$

where $\Delta H_{\text {ovr }}$ is the heat of the overall reaction, and $\mathrm{BE}_{\mathrm{A}}$ and $\mathrm{BE}_{\mathrm{B}}$ are the enthalpy change of sorption of species $\mathrm{A}$ and $\mathrm{B}$, respectively (e.g. $\mathrm{BE}_{\mathrm{A}}=H_{\mathrm{A}}+H_{*}-H_{\mathrm{A}^{*}}$ ). The definition in eq. (19) is such that at $\mathrm{BE}_{\mathrm{A}}=\delta$, the surface reaction becomes isothermic $\left(H_{\mathrm{A}^{*}}=H_{\mathrm{B}^{*}}\right)$, and the change in binding energy of $\mathrm{A}$ and $\mathrm{B}$ are related by $\gamma \Delta \mathrm{BE}_{\mathrm{A}}=\Delta \mathrm{BE}_{\mathrm{B}}$. In this work, we reproduce a previously published example where $\gamma=0.5, \delta=1.4 \mathrm{eV}, \Delta H_{\mathrm{ovr}}=0 \mathrm{eV}$, and the binding energy $\mathrm{BE}_{\mathrm{B}}$ is oscillated from 0.1 to $1.03 \mathrm{eV}$. The activation energy of the surface reaction is (eq. (20)):

$$
E_{\mathrm{a}, \mathrm{sr}}=\alpha \Delta H_{\mathrm{sr}}+\beta
$$

where $\Delta H_{\mathrm{sr}}$ is the enthalpy change of the surface reaction ( $\mathrm{A}^{*}$ to $\left.\mathrm{B}^{*}\right)$, and in this example $\alpha=0.6$ and $\beta=102 \mathrm{~kJ} / \mathrm{mol}$. Following the methodology described above, we reproduce the simulation reported by Ardagh et al. (14) for a square wave $(n=2)$ oscillation in Figure 5a, with excellent agreement between most data points. The discrepancy for frequencies $10^{-2}-10^{-4} \mathrm{~Hz}$ may be because Ardagh et al. (14) simulated a continuous stirred tank reactor where the chemical activities of the 
reactants and products are not fixed in time and the yields may vary slightly from simulation to simulation. Here, no assumption on a reactor configuration is made and rates are reported for fixed activities of reactants and products. A final difference between the simulation here and the simulation from Ardagh et al. (14) is that we capped the value of $k_{-1}$ to $10^{25} \mathrm{~s}^{-1}$, whereas the value from the simulation by Ardagh et al. (14) reached $10^{29} \mathrm{~s}^{-1}$. We capped this value because poor scaling of matrices $\boldsymbol{A}^{[j]}$ or $\boldsymbol{M}$ can cause them to be singular within the numerical precision of Matlab. We also note that the rate constant $k_{-1}>10^{13} \mathrm{~s}^{-1}$ is nonphysical and occurs because the desorption of $\mathrm{A}^{*}$ was given a negative activation energy at some conditions. This has no impact on the theoretical insights of the simulations, and we expect allowing the BEP trends to continue to artificial, or non-physical, regimes is preferred to capping rate constants if one aims to develop theoretical insights regarding the general consequences of BEP relationships in dynamic catalysis. Observed dynamic catalysis behavior, even for artificial rate constants, may be edifying and relevant for some different, more physically realistic choice of $\beta, \delta$, and reference state. We do not believe that adjusting $k_{-1}$ had a significant impact on the comparison of our results to Ardagh et al. (14).

Figure $5 \mathrm{~b}$ shows the computational time per dynamic steady-state calculation as a function of the number of steps in the square wave. For the $n=2$ square wave, each limit cycle takes on average $0.14 \mathrm{~ms}$ to calculate, regardless of the oscillation frequency. This is more than 8 orders of magnitude faster than the high frequency calculations reported by Ardagh et al. (14) using numerical forward integration. Figure 5 also shows the average rate at dynamic steady-state when oscillating binding energies as a sinusoidal wave approximated by an $n$-stepped square wave with varying frequencies. For $n=20$ steps, the CPU time is $\sim 1 \mathrm{~ms}$ per dynamic steady-state calculation and the average rate as a function of frequency already closely resembles the $n=1,000$ steps 
solution, with a difference of $20 \%$ at the maximum rate. On a logarithmic scale, $n=100$ and $n=$ 1,000 steps are not visually discernible but have a maximum deviation of $1.2 \%$. The $n=200$ solution has a maximum deviation from $n=1,000$ of $0.3 \%$, which we regard as an accurate approximation of the continuous sinusoidal wave. Approximating the sine wave with $n=200$ steps only has a median CPU time of $\sim 30 \mathrm{~ms}$ per dynamic steady-state calculation, regardless of the oscillation frequency, and compares well even with solutions calculated for $n=10,000$ at $10^{6} \mathrm{~Hz}$, as is shown in Figure 6a. The general shapes of the two solutions are the same, but the $n=10,000$ solution is smooth while the $n=200$ solution exhibits visible features where the steps occur, as highlighted in Figure 6b. In general, we conclude that the $n=200$ is an excellent approximation of the exact dynamic solution.
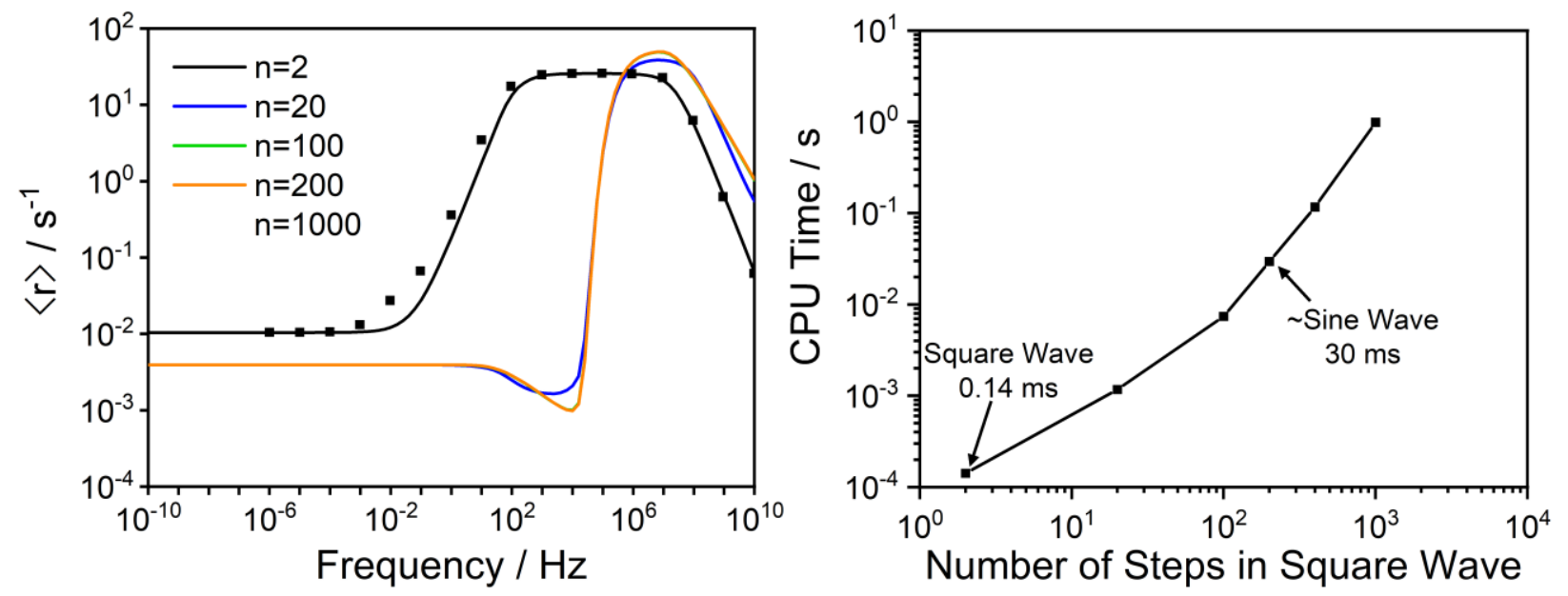

Figure 5. (a) Time-averaged rate as a function of frequency for a sine wave approximated by a stepped-square wave with $n=2-1,000$ steps. The black squares are the time-averaged rates reported by Ardagh et al. (14) for a square wave with $n=2$. (b) The median computational time as a function of square wave steps for the time-averaged rate at dynamic steady state for frequencies ranging from $10^{-10}$ to $10^{10} \mathrm{~Hz}$. A continuous sine wave is well approximated by $n=200$ steps with a median computation time of $30 \mathrm{~ms}$. 

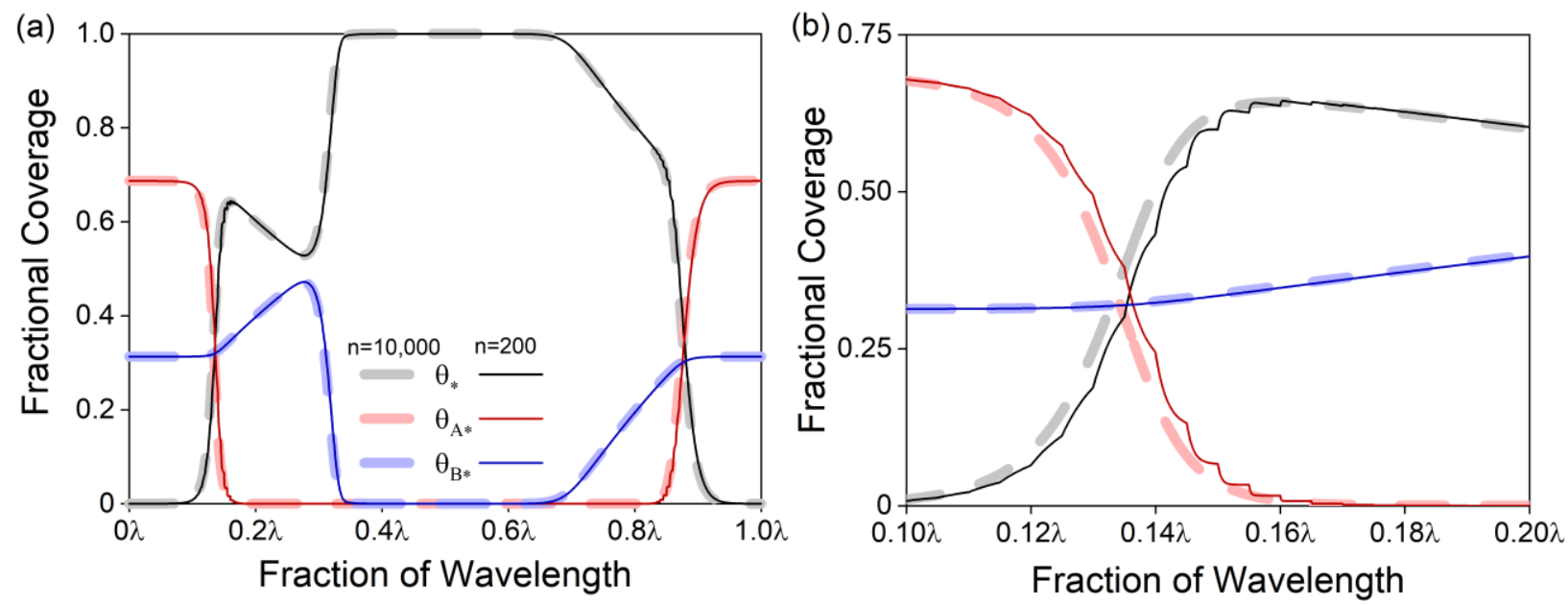

Figure 6. (a) The limit cycle for the sine wave oscillations approximated by $n=200$ (solid lines) and $n=10,000$ (dashed lines) $n$-stepped square waves. The two solutions nearly overlay. (b) A closer look at the two solutions from $t=0.1 \lambda$ to $0.2 \lambda$ shows the bumps that appear in the $n=200$ solution while the $n=10,000$ solution is smooth.

Scheme 2. Linear three-step reaction network

\begin{tabular}{c|c}
\hline $\mathrm{A}+* \rightleftarrows \mathrm{A}^{*}$ & $k_{1}, k_{-1}$ \\
$\mathrm{~A}^{*} \rightleftarrows \mathrm{B}^{*}$ & $k_{2}, k_{-2}$ \\
$\mathrm{~B}^{*} \rightleftarrows \mathrm{B}+*$ & $k_{3}, k_{-3}$ \\
\hline Overall: $\mathrm{A} \Rightarrow \mathrm{B}$ & \\
\hline
\end{tabular}

The presented computationally-efficient method for finding the limit cycles in dynamic catalysis vastly expands the explorable parameter space and thereby facilitates rapid discovery of kinetic regimes and the kinetic/energetic parameters that determine their optimality and delineation. For example, there are four parameters that describe a simple square wave: the binding energy of $\mathrm{B}$ in each kinetic state, $\mathrm{BE} \mathrm{B}_{\mathrm{B}}^{[j]}$, and the time spent at each kinetic state, $\delta t^{[j]}$. Employing the developed formalism, we facilely explore the effect of asymmetric square waveforms (i.e. $\delta t^{[1]} \neq \delta t^{[2]}$ ) in Figure $7 \mathrm{a}$ at the binding energies reported in Figure 5a. Figure $7 \mathrm{a}$ demonstrates that, by introducing asymmetry, the time-averaged rate is increased by a factor of 
two, and that the line $\delta t^{[1]}=\delta t^{[2]}$ corresponding to symmetric oscillations is an edge on a larger "resonance region." Further improvement to rate could be made by brute force testing each parameter of the square wave, but we instead continue to leverage the descriptive potence and computational efficiency proffered of algorithmic methods by treating the discovery of maximum time-averaged rate as an optimization problem.

The objective function to maximize the time-averaged rate is written as eq. (21), where $\langle r\rangle$ is a function of the vector containing the times and binding energies for each kinetic state:

$$
\max \langle r\rangle(\boldsymbol{x}) \text { where } \boldsymbol{x}=\left[\delta t^{[1]}, \delta t^{[2]}, \mathrm{BE}_{\mathrm{B}}^{[1]}, \mathrm{BE}_{\mathrm{B}}^{[2]}\right]^{\mathrm{T}}
$$

This optimization problem is solvable by the method of gradient ascent, which computes the gradient of the time-averaged rate at the current guess, $\left.\boldsymbol{\nabla}\langle r\rangle\right|_{\boldsymbol{x}_{n}}$, and calculates the next guess, $\boldsymbol{x}_{n+1}$, until a convergence criteria is satisfied (eq. (22)):

$$
\boldsymbol{x}_{n+1}=\boldsymbol{x}_{n}+\left.\varepsilon \boldsymbol{\nabla}\langle r\rangle\right|_{x_{n}}
$$

where $\varepsilon$ is a small parameter that controls the step size. We instead utilized Matlab optimization function fminunc which uses the BFGS quasi-Newton method with a cubic line search procedure to find the optimal square wave for the three-step reaction for $0.1<\mathrm{BE}_{\mathrm{B}}<1.03 \mathrm{eV}$. This method converges to a local maximum in $0.1 \mathrm{~s}$ for the initial guess $\delta t^{[1]}=\delta t^{[2]}=0.5 \times 10^{-3} \mathrm{~s}, \mathrm{BE}_{\mathrm{B}}^{[1]}=$ $0.1 \mathrm{eV}, \mathrm{BE}_{\mathrm{B}}^{[2]}=0.8 \mathrm{eV}$. The optimal square wave is depicted in Figure 8a, where the optimal wave stays at a $\mathrm{BE}_{\mathrm{B}}$ of $0.1 \mathrm{eV}$ for $10^{-4} \lambda$, and at $0.9 \mathrm{eV}$ for $0.9999 \lambda$, where $f=1 / \lambda=6.1 \mathrm{MHz}$, and gives $\langle r\rangle=382 \mathrm{~s}^{-1}$, a $\sim 14 \times$ improvement on the maximum for a symmetric square wave with $\mathrm{BE}_{\mathrm{B}}^{[1]}=0.1 \mathrm{eV}$ and $\mathrm{BE}_{\mathrm{B}}^{[1]}=1.03 \mathrm{eV}$ (Figure 5). Repeating the same optimization but with a 20- 
step square wave gives the same solution, suggesting that this asymmetric two-stepped square wave is near the global optimum for these kinetics and constraints.

The fractional coverages of each species during the algorithmically optimized limit cycle is shown in Figure 8b, and the instantaneous rates are shown in Figure 8c. In the optimal square wave, the binding energy of $\mathrm{B}$ is decreased momentarily to rapidly remove all $\mathrm{A}^{*}$ and $\mathrm{B}^{*}$, emptying the surface. The next state of the square wave has a high binding energy of $\mathrm{B}$ to accumulate $\mathrm{A}^{*}$ on the surface and convert the $\mathrm{A}^{*}$ to $\mathrm{B}^{*}$. During this phase, the rate of $\mathrm{B}$ formation is negative as B adsorbs on the catalyst surface from the fluid phase. The negative rate of B formation in the second state is compensated by asymmetry in the square wave which maximizes the time-averaged reaction rate by ensuring time is not needlessly spent during either the accumulation or recovery of surface-bound intermediates-which, for this particular system, corresponds to $10^{4} \times$ more time spent in the accumulation phase. The critical importance of such asymmetry is explicated by the contour plot in Figure $7 \mathrm{~b}$ which, along with Figure 7a, illustrates the parameters which define the resonance region. In both figures, the resonance region is bounded to the right by the ratio $\delta t^{[2]} / \delta t^{[1]}=1$ and to the left by another ratio, $\delta t^{[2]} / \delta t^{[1]}$, which depends on the kinetics of the system. The bottom and top of the resonance region are bound by two of the eigenvalues of the system, in this case, the eigenvalues from kinetic state $2, \lambda_{1}^{[2]}$ and $\lambda_{2}^{[2]}$. These eigenvalues bound the characteristic resonance frequencies of the system and exemplify the physical and mathematical detail conferred by formulating the analysis of dynamic catalysis in terms of the well-established relationship between linear algebra and ordinary differential equations ubiquitous in the description of catalytic reactions. 


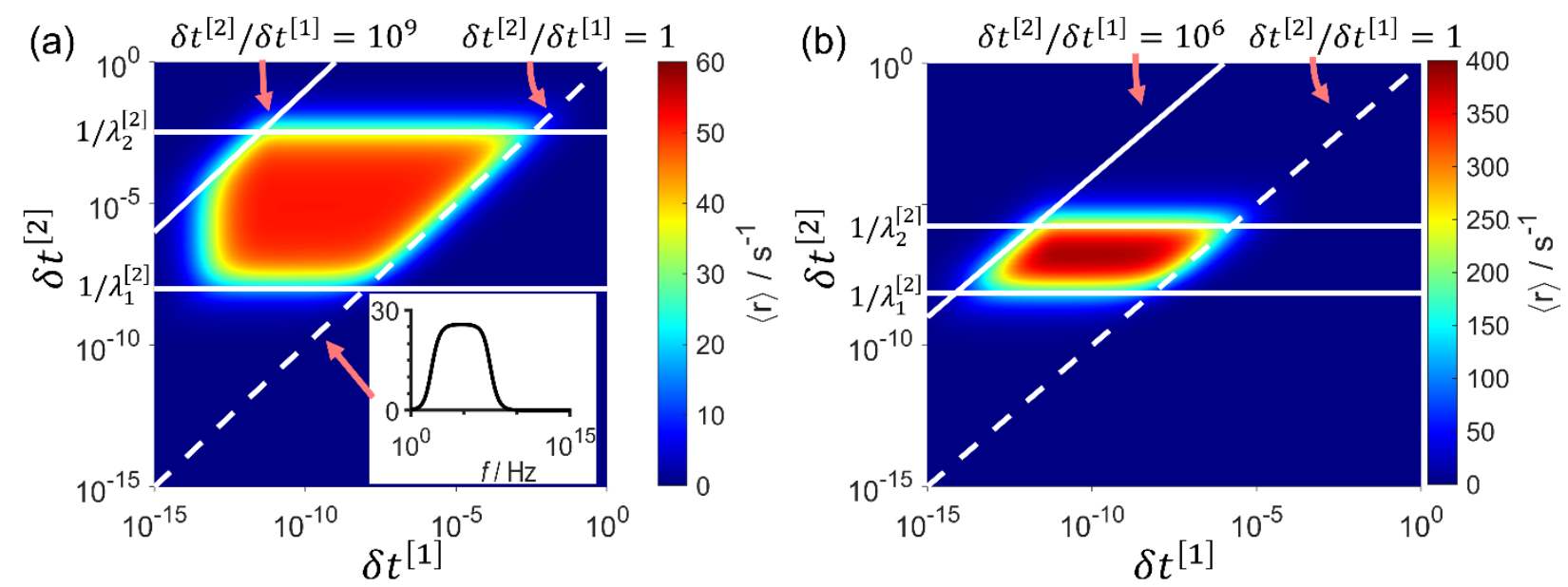

Figure 7. (a) Effect of asymmetric times in a square wave where $\delta t^{[1]}$ is the amount of time spent at the condition $\mathrm{BE}_{\mathrm{B}}=0.1 \mathrm{eV}$ and $\delta t^{[2]}$ is the amount of time at the condition $\mathrm{BE}_{\mathrm{B}}=1.03 \mathrm{eV}$. Maximum rate is $\sim 52 \mathrm{~s}^{-1}$. Inset: Rate as a function of frequency for a symmetric oscillation, which is a diagonal slice of the contour plot. (b) Rate as a function of $\delta t^{[1]}$ and $\delta t^{[2]}$ with $\mathrm{BE}_{\mathrm{B}}^{[1]}=0.1 \mathrm{eV}$ and $\mathrm{BE}_{\mathrm{B}}^{[2]}=0.9 \mathrm{eV}$. Maximum rate at these conditions is $\sim 382 \mathrm{~s}^{-1}$. The upper and lower bounds on the resonance region are determined by the eigenvalues $\lambda_{1}^{[2]}$ and $\lambda_{2}^{[2]}$ (solid white lines), the right bound (white dashed line) corresponds to symmetric oscillation, $\delta t^{[2]}=\delta t^{[1]}$, and the left bound is another line that depends on the kinetics, but corresponds to a constant $\delta t^{[2]} / \delta t^{[1]}$ ratio.

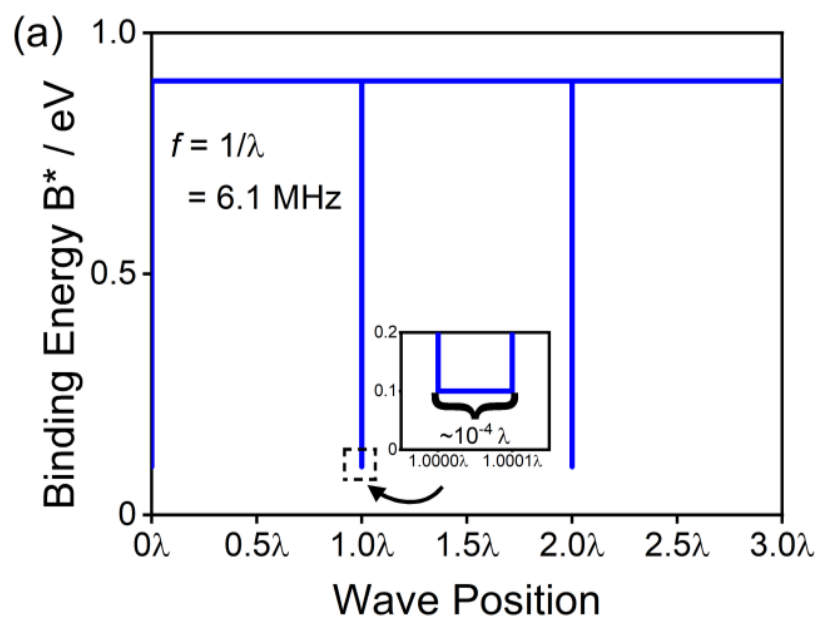



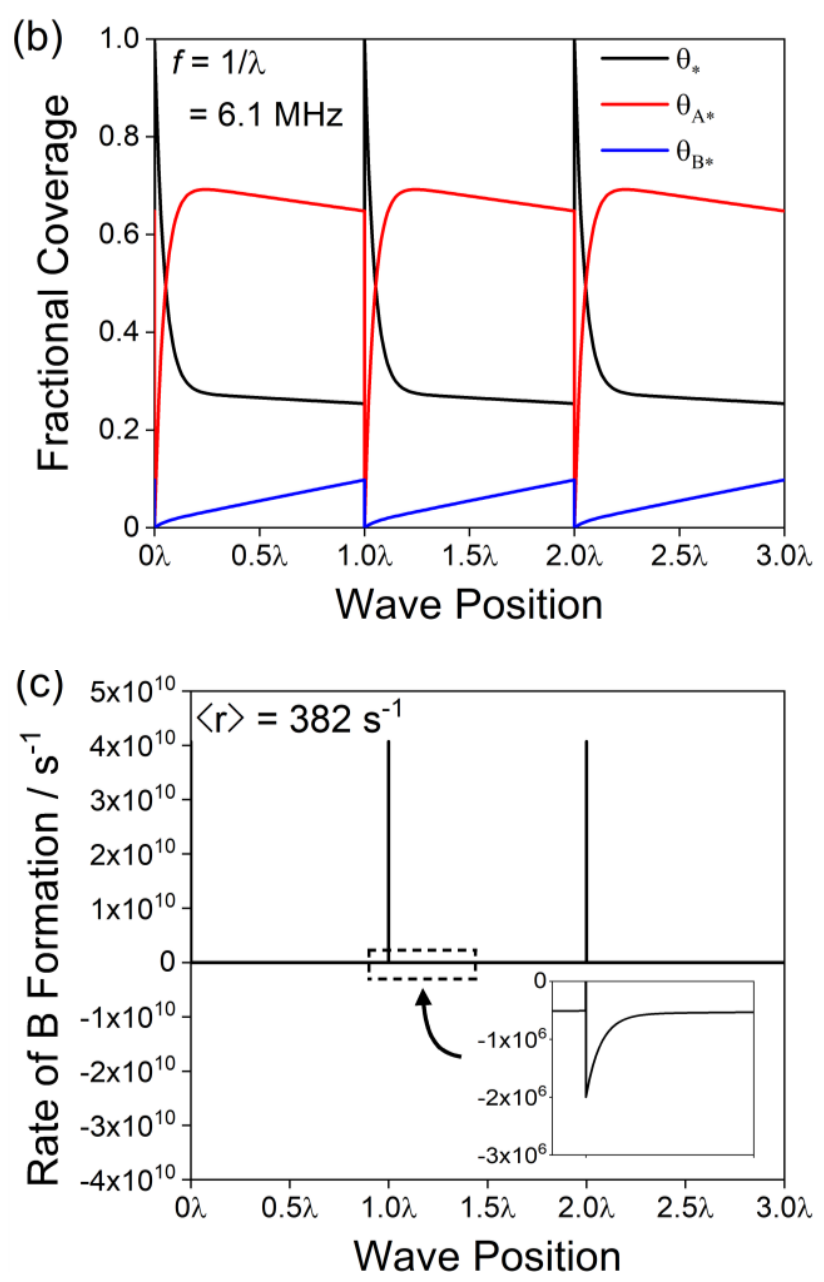

Figure 8. (a) The optimal square wave for the conversion of A to B for the reaction in Scheme 2 as determined by the method of gradient ascent. (b) The fractional coverages of each species during the limit cycle. (c) The rate of B formation during the limit cycle. Inset: A closer inspection of the rate from 0.9 to $1.5 \lambda$. The rate is negative during most of the limit cycle as the high binding energies of $\mathrm{B}$ encourage sorption from the fluid phase.

Thus far, we have described the methods for finding the solution that has the same wavelength as the oscillation $(\lambda)$, but the question remains as to whether this solution is unique. Proofs regarding the criteria for the existence and uniqueness of solutions to first-order differential equations with periodic boundary conditions are present in the literature $(15,16)$, but we present here a logical argument for the existence and uniqueness of the solution to the periodic boundary value problem of the coupled first-order differential equations that arise in dynamic catalysis. 
Equation (12) is a linear coupled ordinary differential equation and has a unique solution for the initial value problem $\boldsymbol{\theta}\left(t=t_{0}\right)=\boldsymbol{\theta}_{\mathbf{0}}$ by the Picard-Lindelöf theorem (17, 18). Thus, if any function is discretized into an infinite-stepped square wave, there exists one unique solution to each step of the square wave for a given initial value. Now, there must be only one initial value that satisfies the periodic boundary problem criteria $\boldsymbol{\theta}(t=0)=\boldsymbol{\theta}(t=\lambda)$ since eqs. (16) and (17) represent a specified system of linear algebraic equations which has only one solution. Because each initial value problem gives a unique solution, and there exists only one initial value vector that satisfies the periodic boundary condition, we conclude that there exists one unique solution to this system of differential equations.

If instead we searched for a solution with a periodicity $n \lambda$, then the periodic boundary condition becomes $\boldsymbol{\theta}(t=0)=\boldsymbol{\theta}(t=n \lambda)$, for which following the same argument as above, there must exist only one unique solution. Further, we know that the solution $\boldsymbol{\theta}(t)$ with periodicity $\lambda$ also satisfies the boundary conditions for any periodicity $n \lambda$, and thus the only periodic solution for linear systems will be those that have the same periodicity as the kinetic oscillation. Proving that aperiodic solutions to this system of equations do not exist is beyond the scope of this work, but this would require the existence of an initial condition $\boldsymbol{\theta}\left(t=t_{0}\right)=\boldsymbol{\theta}_{\mathbf{0}}$ such that $\lim _{n \rightarrow \infty} \boldsymbol{\theta}(t=n \lambda) \neq \boldsymbol{\theta}(t=(n+1) \lambda)$, which does not seem possible for this linear system of equations.

The method described above can be employed to find the limit cycles for any dynamic kinetic system where no reactions occur between species that change in time. However, in nondifferential reactors, the activities of fluid-phase species may be transient, and many important reactions involve the reaction between two surface species, and thus require an alternative method to find the dynamic steady states. Further, as the arguments of the existence of unique periodic 
solutions above required the system of differential equations to be linear, nonlinear differential equations may allow for the possibility of multiple dynamic steady-state solutions, as discussed hereinafter.

\subsection{Finding limit cycle solutions for non-linear reaction systems}

The dynamic steady-state with periodicity $\lambda$ is the solution to systems of differential equations where the periodic boundary condition is satisfied (eq. (23)):

$$
\boldsymbol{\theta}\left(t_{0}\right)=\boldsymbol{\theta}\left(t_{0}+\lambda\right)
$$

We define a function, $F$, that integrates the differential equations over one wavelength, $\lambda$, to give the output $\boldsymbol{\theta}\left(t_{0}+\lambda\right)$ for the initial condition, $\boldsymbol{\theta}\left(t_{0}\right)$, such that (eq. (24)):

$$
\boldsymbol{\theta}\left(t_{0}+\lambda\right)=F\left(\boldsymbol{\theta}\left(t_{0}\right)\right)
$$

After substitution of eq. (24) into eq. (23), our periodic boundary condition becomes (eq. (25)):

$$
\boldsymbol{\theta}=F(\boldsymbol{\theta})
$$

Thus, to satisfy the periodic boundary condition, we need to find the fractional coverages vector, $\boldsymbol{\theta}$, that outputs the same vector $\boldsymbol{\theta}$ after forward integration of one wavelength (function $F$ ). One method for finding this vector is simply by forward integration until a dynamic steady-state is reached, where we guess a vector $\boldsymbol{\theta}^{k}$, and define $\boldsymbol{\theta}^{k+1}=F\left(\boldsymbol{\theta}^{k}\right)$ where $\boldsymbol{\theta}^{k+1}$ is the next guess, and iterate until $\boldsymbol{\theta}^{k+1} \approx \boldsymbol{\theta}^{k}$ is sufficiently satisfied. The efficiency of this algorithm decreases with increasing frequency, for which the method requires forward integration of an indeterminately large number of wavelengths before the periodic boundary condition criteria are satisfied. An alternative approach is using the multivariate Newton-Raphson method, which uses the Jacobian, $J$, to determine the next initial guess. This method involves first defining a function that we wish to minimize. For a periodic boundary condition this can be defined as minimizing the sum of the 
square differences between the input and the output of function $F$ for each surface species $i$ (eq. (26)):

$$
\min \sum_{i} g_{i}\left(\boldsymbol{\theta}^{k}\right)=\sum_{i}\left(\theta_{i}^{k}-F_{i}\left(\boldsymbol{\theta}^{k}\right)\right)^{2}
$$

The Jacobian for the vector function $\boldsymbol{g}\left(\boldsymbol{\theta}^{k}\right)$ is given as (eq. (27)):

$$
\boldsymbol{J}=\left[\begin{array}{ccc}
\frac{\partial g_{1}}{\partial \theta_{1}^{k}} & \cdots & \frac{\partial g_{1}}{\partial \theta_{n}^{k}} \\
\vdots & \ddots & \vdots \\
\frac{\partial g_{n}}{\partial \theta_{1}^{k}} & \cdots & \frac{\partial g_{n}}{\partial \theta_{n}^{k}}
\end{array}\right]
$$

and describes how the function that is being minimized changes with respect to each fractional coverage. The next guess in the Newton-Raphson method is therefore given by:

$$
\boldsymbol{\theta}^{k+1}=\boldsymbol{\theta}^{k}-\boldsymbol{J}^{-1} \boldsymbol{g}\left(\boldsymbol{\theta}^{k}\right)
$$

such that information provided by the Jacobian guides and accelerates the iterative search for the dynamic steady-state coverages. The process is iterated until an arbitrary criterion $\sum_{i} g_{i}\left(\boldsymbol{\theta}^{k}\right)<\varepsilon$ is satisfied. This is one of many methods for finding the local minimum of a function, and other methods may have faster convergence to the local minimum; the primary development of the presented methodology is to reformulate the periodic boundary condition as an optimization problem (eq. (26)), for which many algorithms can be employed to efficiently find the dynamic steady state at high oscillation frequencies.

We demonstrate the computational speed of the Newton-Raphson method for finding the dynamic steady state by considering the reaction network in Scheme 3 . This reaction network is nonlinear because step 3 involves the reaction between two species that are changing in time, $\mathrm{A}^{*}$ and $\mathrm{B}^{*}$, and thus the differential equations are themselves nonlinear. In this example, we consider 
the oscillation of rate constants as simple square waves between two states $j=1$ and $j=2$, with rate constants for each state given in Table 1. The difference between the two kinetic states lies in the affinity of the catalyst to adsorb $A$ and $B$, where kinetic state 1 adsorbs B and ejects $A^{*}$ off the surface, while kinetic state 2 does the opposite.

The convergence of the Newton-Raphson and forward integration methods to the limit cycles are compared in Figure 9 for a frequency $f=10^{2} \mathrm{~Hz}$. The Newton-Raphson method converges to the fractional coverage of $\mathrm{A}^{*}$ at the periodic boundary of the limit cycle, $\theta_{\mathrm{A}^{*}, 0}$, in 11 iteration steps and 1.45 seconds. Forward integration requires more than 100,000 iterations to reach the same value and takes over 2,000 seconds. The computation times of the two methods are compared across decades of oscillation frequency in Figure 9b. At low frequencies, forward integrations will converge to limit cycles in as little as one oscillation, and thus can be faster than the Newton-Raphson method, which requires the numerical calculation of the Jacobian and may take smaller steps in the low frequency regime. At increasing frequencies, the Newton-Raphson method becomes faster because each integration is over a shorter length of time, while the forward integration method generally becomes slower because more oscillations are required before converging to the limit cycle. The decrease in computation time for the forward integration method at $10^{2} \mathrm{~Hz}$ is a consequence of changing chemical dynamics, which decreases the total time required before converging to a limit cycle. 
Scheme 3. Non-linear reaction network

\begin{tabular}{c|c}
\hline $\mathrm{A}+* \rightleftarrows \mathrm{A}^{*}$ & $k_{1}, k_{-1}$ \\
$\mathrm{~B}+* \rightleftarrows \mathrm{B}^{*}$ & $k_{2}, k_{-2}$ \\
$\mathrm{~A}^{*}+\mathrm{B}^{*} \rightleftarrows \mathrm{C}^{*}+*$ & $k_{3}, k_{-3}$ \\
$\mathrm{C}^{*} \rightleftarrows \mathrm{C}+*$ & $k_{4}, k_{-4}$ \\
\hline Overall: $\mathrm{A}+\mathrm{B} \Rightarrow \mathrm{C}$ & \\
\hline
\end{tabular}

Table 1. The hypothetical rate constants for two kinetic states for the reaction sequence in Scheme 3.

\begin{tabular}{l|cc|c|cc}
\hline & $j=1$ & $j=2$ & & $j=1$ & $j=2$ \\
\hline$k_{1}^{[j]}$ & $10^{-5}$ & $10^{5}$ & $k_{-1}^{[j]}$ & $10^{-5}$ & $10^{1}$ \\
$k_{2}^{[j]}$ & $10^{5}$ & $10^{-5}$ & $k_{-2}^{[j]}$ & $10^{1}$ & $10^{-5}$ \\
$k_{3}^{[j]}$ & $10^{1}$ & $10^{1}$ & $k_{-3}^{[j]}$ & $10^{-1}$ & $10^{-1}$ \\
$k_{4}^{[j]}$ & $10^{3}$ & $10^{3}$ & $k_{-4}^{[j]}$ & $10^{-1}$ & $10^{-1}$ \\
\hline
\end{tabular}
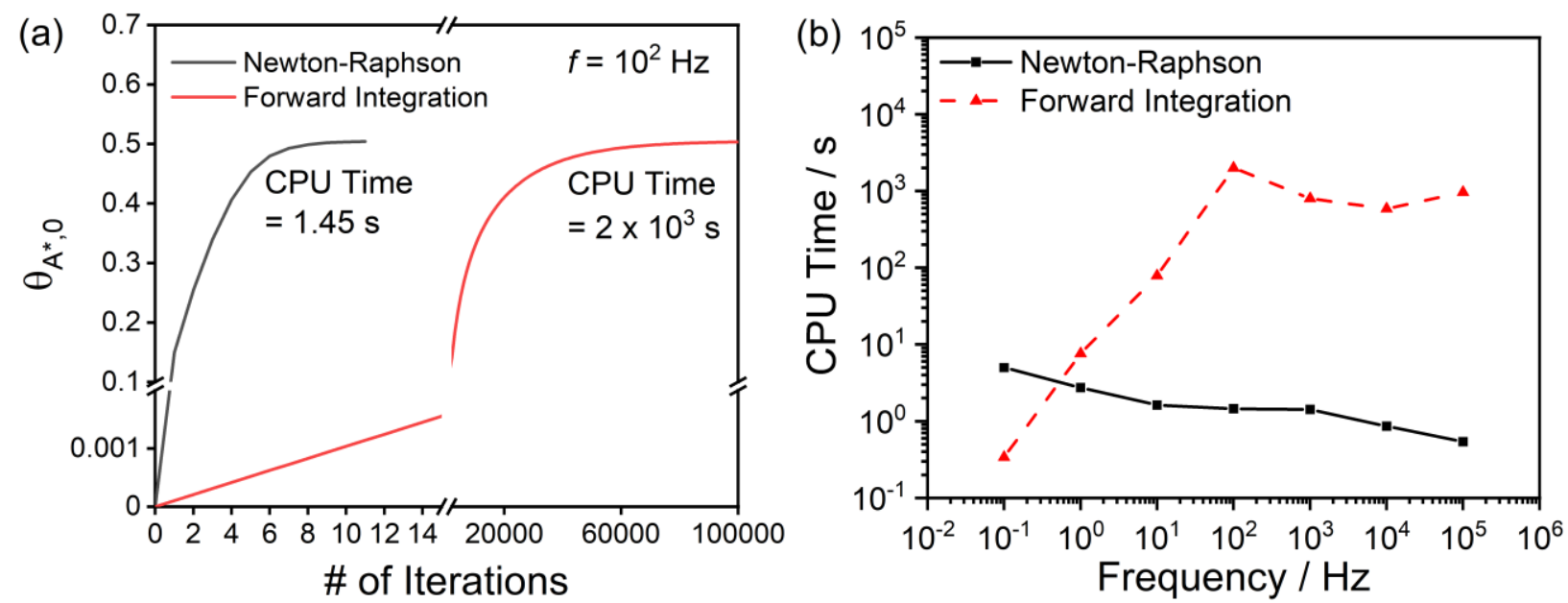

Figure 9. (a) Comparison of the convergence of the Newton-Raphson method to forward integration using relative and absolute tolerances of $10^{-12}$ for the built-in Matlab ${ }^{\circledR}$ solver ode23s. 
(b) Computation time comparison for Newton-Raphson and forward integration as a function of the frequency. Limit cycle solutions were calculated from an initial guess of $\theta_{*}=1$.

For nonlinear reaction systems, such as the network shown in Scheme 3 or for ammonia synthesis $(13,19)$, it is unclear whether one or multiple solutions exist for the periodic boundary value problem. Using a mixture of the Newton-Raphson method and forward integration, the fractional coverage of $\mathrm{A}^{*}$ at the periodic boundary, $\theta_{\mathrm{A}^{*}, 0}$, was found as a function of the squarewave oscillation frequency, as shown in Figure 10. At the limits of low and high frequencies, there was only one limit-cycle solution. However, at intermediate frequencies of $10^{-1}$ to $10^{2} \mathrm{~Hz}$, three limit-cycle solutions were found, one of which was unstable and diverges with any slight perturbation. These unstable limit cycles require the Newton-Raphson solver, because unstable solutions are located at saddle points that locally minimize the criterion in eq. (26), but can fundamentally never be reached by forward integration. The fractional coverage of $A^{*}$ in the stable (solid) and unstable (dashed) limit cycles are shown in Figure 11; at all conditions, the fractional coverages of $\theta_{*}$ and $\theta_{\mathrm{C}^{*}}$ are near zero, and thus the fractional coverage $\theta_{\mathrm{B}^{*}}(t) \approx 1-\theta_{\mathrm{A}^{*}}(t)$.
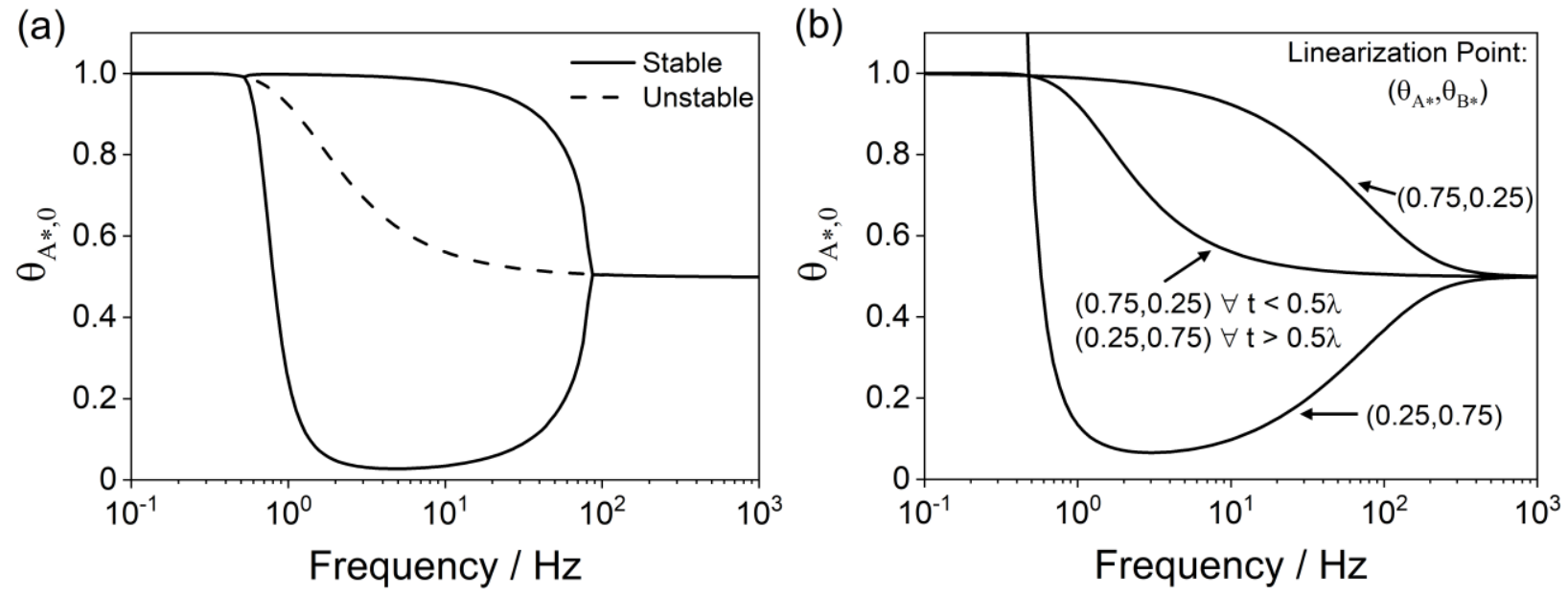

Figure 10. (a) The fractional coverage of $A^{*}$ for limit cycle solutions at the start of a square-wave oscillation (the beginning of kinetic state $j=1$ ) for the reaction sequence and kinetics in Scheme 3 and Table 1, respectively. The solid lines are stable solutions and the dashed line is the unstable solution. (b) An approximation made by linearizing the differential equations about varying surface coverages. Linearization points are shown in parantheses for $\theta_{\mathrm{A}^{*}}$ and $\theta_{\mathrm{B}^{*}}$. The linearization 
points for $\theta_{*}$ and $\theta_{\mathrm{C}^{*}}$ were both zero. The middle curve used a different linearization point for each kinetic state.
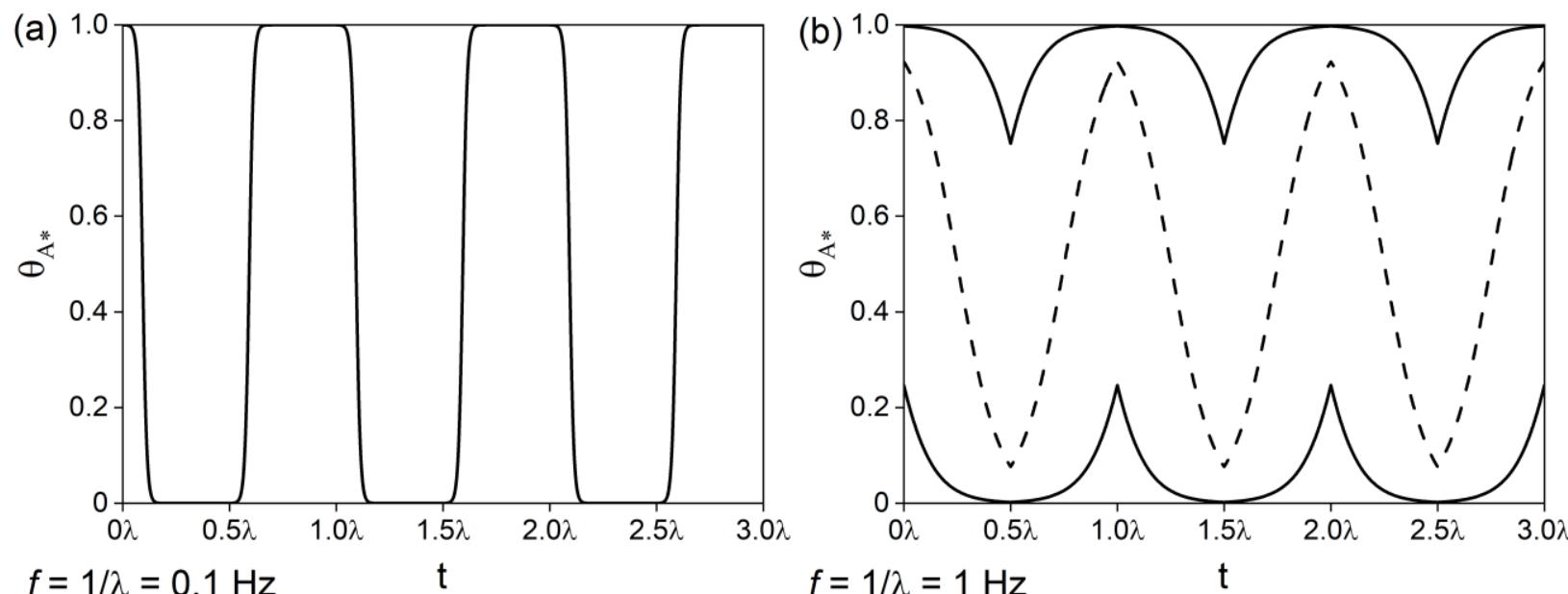

$f=1 / \lambda=0.1 \mathrm{~Hz}$

$f=1 / \lambda=1 \mathrm{~Hz}$
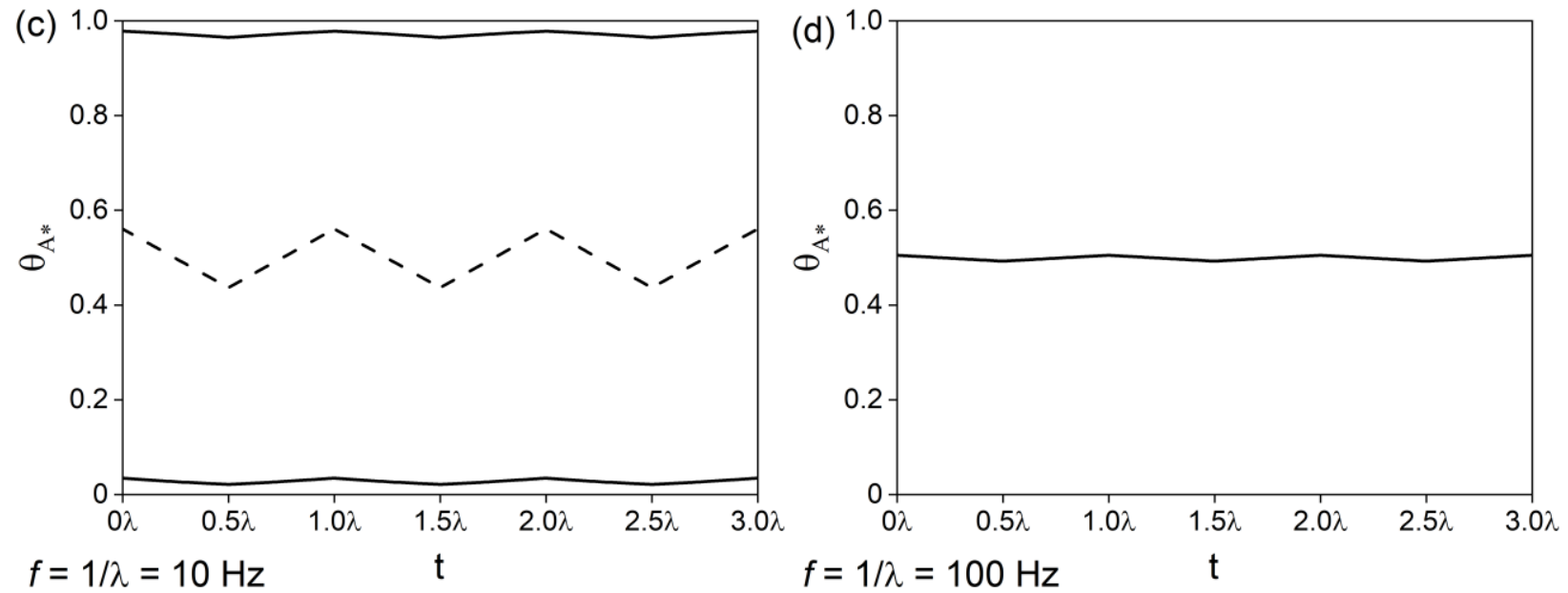

$f=1 / \lambda=10 \mathrm{~Hz}$

$\mathrm{t}$

$f=1 / \lambda=100 \mathrm{~Hz}$

Figure 11. The limit cycles for the fractional coverage of $\mathrm{A}^{*}$ at frequencies of (a) $0.1 \mathrm{~Hz}$, (b) $1 \mathrm{~Hz}$, (c) $10 \mathrm{~Hz}$, and (d) $100 \mathrm{~Hz}$ for reaction network in Scheme 3 with square wave oscillation kinetics between states $j=1$ and $j=2$ in Table 1 . Solid lines are stable limit cycles. Dashed lines are unstable limit cycles. Only limit cycles that satisfy the periodic boundary condition $\boldsymbol{\theta}(t=0)=$ $\boldsymbol{\theta}(t=\lambda)$ were considered.

In Figure 10, the limiting behaviors at high and low frequencies are connected smoothly by the unstable states, while the stable states diverge sharply at the onset of instability. The unstable states have the property that $\theta_{\mathrm{B}^{*}}(t) \approx 1-\theta_{\mathrm{A}^{*}}(t) \approx \theta_{\mathrm{A}^{*}}(t-1 / 2 \lambda)$, and thus the fractional coverages $\theta_{\mathrm{A}^{*}}$ and $\theta_{\mathrm{B}^{*}}$ oscillate symmetrically about $\sim 0.5$. This behavior is stable at the limit of low and high frequencies but becomes unstable at intermediate frequencies. At low frequencies, 
the oscillation frequency is sufficiently small that the catalyst surface essentially reaches static steady-state in each oscillation, reaching the bounds of $\theta_{\mathrm{A}^{*}} \approx 0$ and $\theta_{\mathrm{A}^{*}} \approx 1$ (Figure 11a). As the frequency increases, the fractional coverages no longer proceed via a sequence of steady states, and the stable solutions diverge at the expense of an unstable limit cycle. At sufficiently large frequencies, the stable solutions separate from the bounds at $\theta_{\mathrm{A}^{*}} \approx 0$ and $\theta_{\mathrm{A}^{*}} \approx 1$ and ultimately converge at the quasi-static surface coverage $\theta_{\mathrm{A}^{*}}(t) \approx 0.5$..

The Newton-Raphson method for finding the limit cycle of nonlinear periodic differential equations can be much faster than forward integration (Figure 9), but is significantly slower than the linear algebra method employed for linear reaction schemes. One method for accelerating the integration of nonlinear differential equations is by Taylor linearization, where the differential equations are linearized by the formula (eq. (29)):

$$
\frac{\mathrm{d} x}{\mathrm{~d} t}=F(x, y) \approx F\left(x_{0}, y_{0}\right)+\left.\frac{\partial F}{\partial x}\right|_{x_{0}, y_{0}}\left(x-x_{0}\right)+\left.\frac{\partial F}{\partial y}\right|_{x_{0}, y_{0}}\left(y-y_{0}\right)
$$

where, for example, $F$ is a function of two variables $x$ and $y$ and is linearized about some point $\left(x_{0}, y_{0}\right)$. Linearizing the differential equation for each reaction intermediate in this way, we obtain a set of linear equations that are analytically solved following eqs. (10)-(17). Doing so decreases the computation time by several orders of magnitude, but will only give one solution, despite the actual differential equations having two stable and one unstable limit cycle. Furthermore, the solution is sensitive to the choice in linearization point, as shown by approximate $\theta_{\mathrm{A}^{*}, 0}$ obtained by linearizing the differential equations for the nonlinear reaction in Scheme 3 with kinetics in Table 1. Choices in linearization points were informed by the true solutions depicted in Figure 11. The linearization approximates the true solutions, but further work is necessary to understand the conditions at which multiple steady states may arise and how to choose reasonable linearization points a priori. 
The existence of multiple limit cycles may be problematic in practical application. First, for the reaction in Scheme 2, the stable solutions give surfaces that are much less evenly distributed between $\mathrm{A}^{*}$ and $\mathrm{B}^{*}$, and thus will have lower rates than the unstable solution. Second, any perturbations in the system may result in jumping from one limit cycle to another, causing unpredictable changes in reaction rate, heat generation, optimal feed composition, and outlet composition — leading to many system controls issues (20). In practice, regimes of multiple steady states are typically best avoided. In general, for nonlinear reaction systems, we cannot determine the number of possible limit cycles during dynamic catalysis, nor is it clear at what frequencies these multiple limit cycles will arise, though they are likely related to the time scales for kinetic processes (e.g., quasi-equilibrium of reaction or quasi-steady-state of species). This problem has many similarities to Hilbert's sixteenth problem, as yet unsolved, which concerns the number of limit cycles that exist for a coupled system of two variables with time-independent polynomial differential equations (21). We can also make no justifiable comment on when solutions with different periodicities or aperiodic, chaotic solutions generally exist under dynamic catalysis conditions; however, we contend that, at the limit of low and high frequencies, there will always be one unique limit cycle solution if the reaction network gives only one static steady-state solution, as we discuss next.

At the low frequency limit, if the reaction network allows for only one steady-state solution under static kinetics, as determined by chemical reaction network theory (22), then there exists only one limit cycle during dynamic kinetics. This conclusion is arrived at by recognizing that for sufficiently low frequencies, sufficient time is spent in each kinetic state such that, for most of the time spent in each state, rates and surface coverages are time-invariant. Thus, at the low frequency limit, the fractional coverages of the surface can be approximated as $\theta_{j^{*}}(t) \approx \theta_{j^{*}, \mathrm{SS}}(t)$, where 
$\theta_{j^{*}, \mathrm{SS}}(t)$ is the steady-state fractional coverage for species $j^{*}$ for the kinetics at time $t$. In this sense, the reaction simply proceeds via a series of static steady states. Therefore, if the reaction network allows for only one steady-state solution during static catalysis, then at each time $t$ there is only one $\theta_{j^{*}, \mathrm{SS}}(t)$ —ensuring that there is only one limit cycle during dynamic catalysis.

The unique limit cycle in the high frequency limit is defined, not by a series of steady states at each condition, but a single quasi-static steady state maintained continuously and repeating each oscillation. In a quasi-static steady state, the oscillation frequency is sufficiently large such that the fractional coverages of species are essentially constant $\left(\theta_{j^{*}}(t) \approx \theta_{j^{*}}\right)$. Therefore, the timeaveraged rate for each elementary step $r_{i}$ is a product of the elementary step rate constant, $k_{i}(t)$, multiplied by a function of time-invariant fractional coverages $F\left(\theta_{j^{*}}(t)\right)$, which is a product of the fractional coverages of species involved in elementary step $i$ in accordance with mass-action constitutive equations (eq. (30)):

$$
\left\langle r_{i}\right\rangle=\frac{\int_{0}^{1 / f} k_{i}(t) F\left(\theta_{j^{*}}(t)\right) \mathrm{d} t}{\int_{0}^{1 / f} \mathrm{~d} t} \stackrel{\lim _{f \rightarrow \infty}}{\approx} F\left(\theta_{j^{*}}\right) \frac{\int_{0}^{1 / f} k_{i}(t) \mathrm{d} t}{\int_{0}^{1 / f} \mathrm{~d} t}=\left\langle k_{i}\right\rangle F\left(\theta_{j^{*}}\right)
$$

Thus, the equations for the time-averaged rates of each elementary step are identical to the equations for static catalysis, with the exception that the rate constants are now time-averaged. The systems of equations that describe the reaction kinetics for a given reaction network under static kinetics and quasi-static surface coverages during dynamic kinetics are structurally identical. Therefore, the number of steady-state solutions for a reaction network during static kinetics must be the equal to the number of limit cycles during dynamic catalysis at the high frequency limit, and the differential equations for fractional coverages are entirely analogous to a static steady-state system (eq. (31)): 


$$
\begin{aligned}
& \frac{\mathrm{d} \theta_{*}}{\mathrm{~d} t} \approx\left\langle k_{-1}\right\rangle \theta_{\mathrm{A}^{*}}-\left\langle k_{1}\right\rangle a_{\mathrm{A}} \theta_{*}+\left\langle k_{-2}\right\rangle \theta_{\mathrm{B}^{*}}-\left\langle k_{2}\right\rangle a_{\mathrm{B}} \theta_{*}+\left\langle k_{3}\right\rangle \theta_{\mathrm{A}^{*}} \theta_{\mathrm{B}^{*}}-\left\langle k_{-3}\right\rangle \theta_{\mathrm{C}^{*}} \theta_{*} \\
&+\left\langle k_{4}\right\rangle \theta_{\mathrm{C}^{*}}-\left\langle k_{-4}\right\rangle a_{\mathrm{C}} \theta_{*} \\
& \frac{\mathrm{d} \theta_{\mathrm{A}^{*}}}{\mathrm{~d} t} \approx-\left\langle k_{-1}\right\rangle \theta_{\mathrm{A}^{*}}+\left\langle k_{1}\right\rangle a_{\mathrm{A}} \theta_{*}-\left\langle k_{3}\right\rangle \theta_{\mathrm{A}^{*}} \theta_{\mathrm{B}^{*}}+\left\langle k_{-3}\right\rangle \theta_{\mathrm{C}^{*}} \theta_{*} \\
& \frac{\mathrm{d} \theta_{\mathrm{B}^{*}}}{\mathrm{~d} t} \approx-\left\langle k_{-2}\right\rangle \theta_{\mathrm{B}^{*}}+\left\langle k_{2}\right\rangle a_{\mathrm{B}} \theta_{*}-\left\langle k_{3}\right\rangle \theta_{\mathrm{A}^{*}} \theta_{\mathrm{B}^{*}}+\left\langle k_{-3}\right\rangle \theta_{\mathrm{C}^{*}} \theta_{*} \\
& \frac{\mathrm{d} \theta_{\mathrm{C}^{*}}}{\mathrm{~d} t} \approx\left\langle k_{3}\right\rangle \theta_{\mathrm{A}^{*}} \theta_{\mathrm{B}^{*}}-\left\langle k_{-3}\right\rangle \theta_{\mathrm{C}^{*}} \theta_{*}-\left\langle k_{4}\right\rangle \theta_{\mathrm{C}^{*}}+\left\langle k_{-4}\right\rangle a_{\mathrm{C}} \theta_{*}
\end{aligned}
$$

The quasi-static surface assumption is an excellent approximation at sufficiently high frequencies, as shown in Figure 12a. At lower frequencies, the quasi-static surface assumption is not rigorously valid over the entire transient, yet can still converge to approximately the same limit cycle, as shown in Figure 12b. This is because for the first oscillation in Figure 12b, the quasi-static surface approximation is not valid as A* quickly covers the surface. During subsequent oscillations, the quasi-static surface approximation becomes valid, which is why they ultimately converge to the same steady state condition.
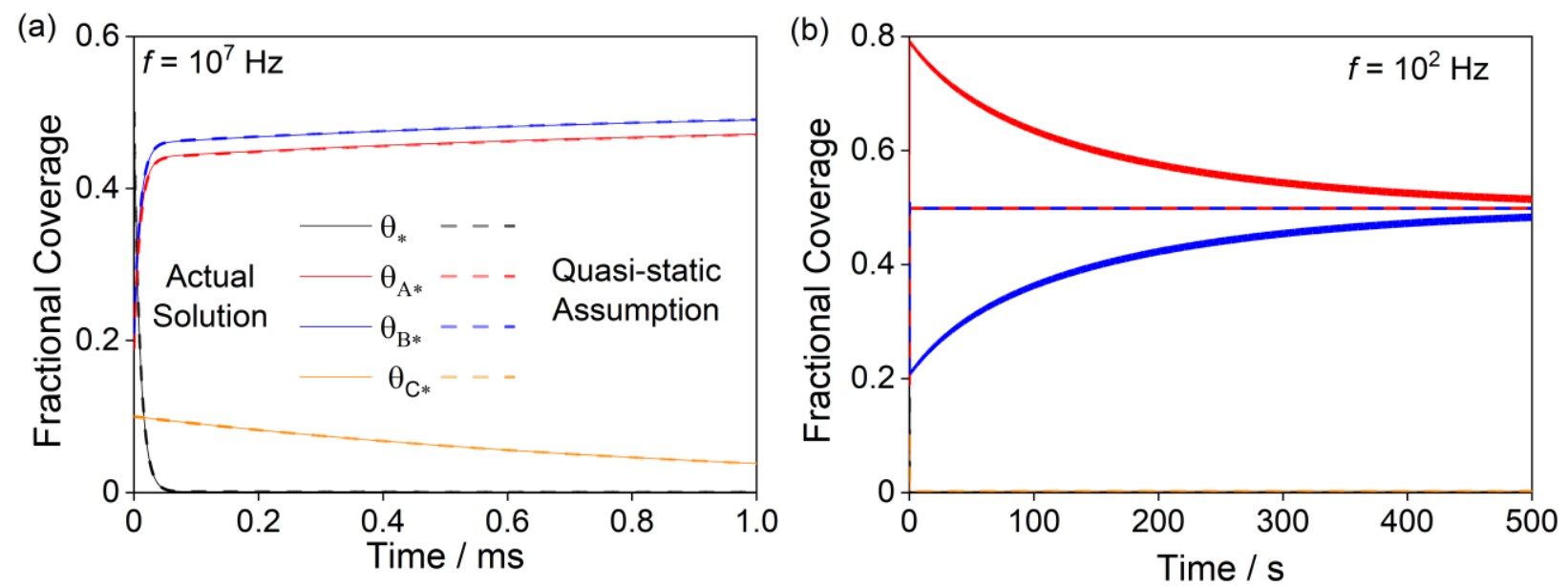

Figure 12. A comparison of the quasi-static surface assumption solution and the transient during dynamic catalysis at symmetric square wave oscillation frequencies of (a) $10^{7} \mathrm{~Hz}$ and (b) $10^{2} \mathrm{~Hz}$. The initial coverage for each simulation was $\theta_{*}=0.5, \theta_{\mathrm{A}^{*}}=0.19, \theta_{\mathrm{B}^{*}}=0.21, \theta_{\mathrm{C}^{*}}=0.1$. The kinetics and reaction network are reported in Scheme 3 and Table 1. 
The quasi-static surface assumption reveals that apparent rate constants of elementary steps can be favorably altered by time-averaging the rate constants of two different kinetic states when the kinetic oscillation frequency is sufficiently large. This confirms that, while resonance certainly can be a factor for enhancing the rate (Figure 7), it is not a necessary pre-condition for enhanced rate, selectivity, or conversion during dynamic catalysis. Instead, as recognized by Astumian and coworkers $(7,8,23)$ the fundamental prerequisite for rate enhancement by dynamic catalysis is kinetic asymmetry between the energetic states through which the catalyst is cycled. Rate enhancement by time-averaging of rate constants at quasi-static surface conditions and by resonance represent two different mechanisms by which dynamic catalysis can enhance rates, selectivities, and conversions. Understanding under which conditions one mechanism is favored is a topic that warrants further research.

\section{Conclusion}

We establish methods significantly faster than numerical forward integration for finding the limit cycles and time-averaged rates for dynamic catalytic systems. These methods calculate the limit cycles for kinetic oscillations of any shape with computation times that are essentially independent of oscillation frequency and enable facile discovery of the optimal kinetic waveform that maximizes the time-averaged reaction rate using optimization methods. The approach for linear systems, where no time-dependent species react with each other, uses linear algebra to analytically solve for the limit cycles. For nonlinear systems, the coupled ODEs and corresponding periodic boundary conditions are recast as criteria in an optimization problem solved by a NewtonRaphson approach. For linear systems, it is shown that there exists only one periodic limit cycle, but for nonlinear systems, multiple limit cycles exist. Generally, if the reaction network allows for 
only one steady-state solution under static kinetic conditions, only one limit cycle exists under dynamic conditions in the limit of low oscillation frequency, for which the reaction proceeds via a series of steady states, and in the limit of high oscillation frequency, for which the reaction is maintained at a single quasi-static state. For intermediate oscillation frequencies, no such simplifying conditions exist, and multiple nonlinear solutions are expected.

Under sufficiently fast kinetic oscillations, the activities of species are "quasi-static" in comparison to the frequency of kinetic oscillations, and thus the reaction network behaves identically to a static reaction network with rate constants that are equal to the time-averaged rate constants of the kinetic waveforms. These conditions are rapidly simulated by forward integration regardless of whether the reaction network is linear. Analysis of reaction networks under quasistatic conditions reveal that resonance is not always a necessary condition to observe enhanced kinetics during dynamic catalysis; rather, the principal requirement for rate enhancement is asymmetry of the kinetic states sampled by the oscillation waveform.

\section{Acknowledgements}

This work was performed in part under the auspices of the U.S. Department of Energy by Lawrence Livermore National Laboratory under Contract DE-AC52-07NA27344.

\section{Supporting Information}

Matlab code is downloadable for free from https: Ilwww.github.com/foley352/dynamic.

\section{Notes}

The authors declare no competing financial interest. 


\section{References}

1. J. Qi, et al., Dynamic Control of Elementary Step Energetics via Pulsed Illumination Enhances Photocatalysis on Metal Nanoparticles. ACS Energy Lett. 5, 3518-3525 (2020).

2. M. Shetty, et al., The Catalytic Mechanics of Dynamic Surfaces: Stimulating Methods for Promoting Catalytic Resonance. ACS Catal. 10, 12666-12695 (2020).

3. D. A. Gonzalez-Casamachin, J. Rivera De la Rosa, C. J. Lucio-Ortiz, L. Sandoval-Rangel, C. D. García, Partial oxidation of 5-hydroxymethylfurfural to 2,5-furandicarboxylic acid using $\mathrm{O}_{2}$ and a photocatalyst of a composite of $\mathrm{ZnO} / \mathrm{PPy}$ under visible-light: Electrochemical characterization and kinetic analysis. Chem. Eng. J. 393, 124699 (2020).

4. F. Sordello, F. Pellegrino, M. Prozzi, C. Minero, V. Maurino, Controlled Periodic Illumination Enhances Hydrogen Production by over $50 \%$ on $\mathrm{Pt} / \mathrm{TiO}_{2}$. ACS Catal. 11, 6484 6488 (2020).

5. J. Gopeesingh, et al., Resonance-Promoted Formic Acid Oxidation via Dynamic Electrocatalytic Modulation. ACS Catal. 10, 9932-9942 (2020).

6. C. Cheng, et al., An artificial molecular pump. Nat. Nanotechnol. 10, 547-553 (2015).

7. R. D. Astumian, et al., Non-equilibrium kinetics and trajectory thermodynamics of synthetic molecular pumps. Mater. Chem. Front 4, 1304 (2020).

8. R. D. Astumian, Kinetic asymmetry allows macromolecular catalysts to drive an information ratchet. Nat. Commun. 10, 3837 (2019).

9. M. A. Ardagh, O. A. Abdelrahman, P. J. Dauenhauer, Principles of Dynamic Heterogeneous Catalysis: Surface Resonance and Turnover Frequency Response. ACS Catal. 9, 6929-6937 (2019).

10. R. D. Astumian, B. Robertson, Imposed Oscillations of Kinetic Barriers Can Cause an Enzyme To Drive a Chemical Reaction Away from Equilibrium. J. Am. Chem. Soc. 115 (1993).

11. R. D. Astumian, I. Derényi, Fluctuation driven transport and models of molecular motors and pumps. Eur. Biophys. J. 27, 474-489 (1998).

12. D. S. Liu, R. D. Astumian, T. Y. Tsong, Activation of $\mathrm{Na}^{+}$and $\mathrm{K}^{+}$pumping modes of (Na,K)-ATPase by an oscillating electric field. J. Biol. Chem. 265, 7260-7267 (1990).

13. G. R. Wittreich, S. Liu, P. J. Dauenhauer, D. G. Vlachos, Catalytic Resonance of Ammonia Synthesis by Dynamic Ruthenium Crystal Strain. ChemRxiv (2021). 
14. M. A. Ardagh, T. Birol, Q. Zhang, O. A. Abdelrahman, P. J. Dauenhauer, Catalytic resonance theory: superVolcanoes, catalytic molecular pumps, and oscillatory steady state. Catal. Sci. Technol. 9, 5058 (2019).

15. C. C. Tisdell, Existence of solutions to first-order periodic boundary value problems. $J$. Math. Anal. Appl. 323, 1325-1332 (2006).

16. S. Boussandel, Existence and uniqueness of periodic solutions for gradient systems in finite dimensional spaces. Acta Math. Sci. 36, 233-243 (2016).

17. E. A. Coddington, N. Levinson, Theory of Ordinary Differential Equations (McGraw-Hill, 1955).

18. D. M. Marquis, E. Guillaume, A. Camillo, T. Rogaume, F. Richard, Existence and uniqueness of solutions of a differential equation system modeling the thermal decomposition of polymer materials. Combust. Flame 160, 818-829 (2013).

19. Á. Logadóttir, J. K. Nørskov, Ammonia synthesis over a Ru(0001) surface studied by density functional calculations. J. Catal. 220, 273-279 (2003).

20. O. Levenspiel, Chemical Reaction Engineering, 3rd Ed. (John Wiley \& Sons, 1999).

21. D. Hilbert, Mathematical problems. Bull. Am. Math. Soc. 8, 437-479 (1902).

22. M. Feinberg, Chemical reaction network structure and the stability of complex isothermal reactors-I. The deficiency zero and deficiency one theorems. Chem. Eng. Sci. 42, 22292268 (1987).

23. R. D. Astumian, P. B. Chock, T. Y. Tsong, Y.-D. Chen, H. V. Westerhofft, Can free energy be transduced from electric noise? Proc. Natl. Acad. Sci. 84, 434-438 (1987). 